\title{
Virtual fatigue diagnostics of wake- affected wind turbine via Gaussian Process Regression
}

\section{Journal Article}

\section{Author(s):}

Avendaño Valencia, Luis David (i); Abdallah, Imad (i); Chatzi, Eleni (i)

Publication date:

2021-06

Permanent link:

https://doi.org/10.3929/ethz-b-000470688

\section{Rights / license:}

Creative Commons Attribution-NonCommercial-NoDerivatives 4.0 International

\section{Originally published in:}

Renewable Energy 170, https://doi.org/10.1016/j.renene.2021.02.003

\section{Funding acknowledgement:}

679843 - Smart Monitoring, Inspection and Life-Cycle Assessment of Wind Turbines (EC)

825833 - An autonomous Real-Time Decision Tree framework for monitoring and diagnostics on wind turbines (EC) 


\title{
Virtual fatigue diagnostics of wake-affected wind turbine via Gaussian Process Regression
}

\author{
Luis David Avendaño-Valencia a, ${ }^{\text {, }}$ Imad Abdallah ${ }^{\text {b, *, }}{ }^{\text {, Eleni Chatzi }}{ }^{\text {b }}$ \\ a Department of Mechanical and Electrical Engineering University of Southern Denmark, Campusvej 55, 5230, Odense M, Denmark \\ ${ }^{\mathrm{b}}$ Institute of Structural Engineering, ETH Zurich, Switzerland
}

\section{A R T I C L E I N F O}

\section{Article history:}

Received 8 November 2020

Received in revised form

31 January 2021

Accepted 1 February 2021

Available online 8 February 2021

\section{Keywords:}

Wind turbine

Fatigue

Wake

Uncertainty

Bayesian Gaussian process regression

Virtual sensing

\begin{abstract}
A B S T R A C T
We propose a data-driven model to predict the short-term fatigue Damage Equivalent Loads (DEL) on a wake-affected wind turbine based on wind field inflow sensors and/or loads sensors deployed on an adjacent up-wind wind turbine. Gaussian Process Regression (GPR) with Bayesian hyperparameters calibration is proposed to obtain a surrogate from input random variables to output DELs in the blades and towers of the up-wind and wake-affected wind turbines. A sensitivity analysis based on the hyperparameters of the GPR and Kullback-Leibler divergence is conducted to assess the effect of different input on the obtained DELs. We provide qualitative recommendations for a minimal set of necessary and sufficient input random variables to minimize the error in the DEL predictions on the wake-affected wind turbine. Extensive simulations are performed comprising different random variables, including wind speed, turbulence intensity, shear exponent and inflow horizontal skewness. Furthermore, we include random variables related to the blades lift and drag coefficients with direct impact on the rotor aerodynamic induction, which governs the evolution and transport of the meandering wake. In addition, different spacing between the wind turbines and Wöhler exponents for calculation of DELs are considered. The maximum prediction normalized mean squared error, obtained in the tower base DELs in the fore-aft direction of the wake affected wind turbine, is less than $4 \%$. In the case of the blade root DELs, the overall prediction error is less than $1 \%$. The proposed scheme promotes utilization of sparse structural monitoring (loads) measurements for improving diagnostics on wake-affected turbines.
\end{abstract}

(C) 2021 The Author(s). Published by Elsevier Ltd. This is an open access article under the CC BY-NC-ND license (http://creativecommons.org/licenses/by-nc-nd/4.0/).

\section{Introduction}

The load effects and degradation of structural components of wind turbines (WTs) do not uniformly evolve across a wind farm due to, largely, wake effects and variability in the inflow conditions (and waves for offshore wind farms [27]). The assessment of fatigue damage accumulation on assumption of availability of direct load effects measurements on all main structural components across all wind turbines within a wind farm is not realistic. The assumption of availability of high-fidelity aero-servo-elastic simulators of the investigated wind turbines coupled to site-specific inflow and wake models is convenient, but often not borne out of the actual reality experienced by wind farm owners and operators. At best, the designer/manufacturer of the wind turbine might make a single

\footnotetext{
* Corresponding author.

E-mail address: abdallah@ibk.baug.ethz.ch (I. Abdallah).

1 Authors made equal contributions to the study and the publication.
}

comprehensive one-off set of output load effects of such wind farm simulations available to the owner/operator of the wind farm.

A further and, from the view of this paper, perhaps more important hindrance lies in the precise estimation of structural response signals that are typically not available in the standard data emanating from the Supervisory Control and Data Acquisition (SCADA) monitoring systems embedded on wind turbines. A number of works therefore attempt condition monitoring on the basis of SCADA availability [28,50]. However, when structural monitoring information becomes available [19], then this can be exploited as a more direct proxy to diagnose sudden damage [8], or to further accurately assess the remaining useful lifetime of a wind turbine in a given wind farm [18,21,36,47]. We therefore posit that considerable improvements to the operation, maintenance and prediction of remaining useful life of a wind turbine can be accomplished by delivering access to transparent, simple, yet powerful and interpretable data-driven predictive models. Such models could be trained, tuned and updated via (fairly) easily accessible and cheap structural response observations from a 


\section{Nomenclature}

$\alpha \quad$ Wind shear exponent

$\mathbb{E} \quad$ Expected value of a random variable

$\mathbb{V} \quad$ Variance of a random variable

$\mathscr{D} \quad$ Rotor diameter

$\Psi \quad$ Inflow horizontal skewness

$\sigma \quad$ Turbulence

$C_{D} \quad$ Aerodynamic drag coefficient

$C_{L} \quad$ Aerodynamic lift coefficient

DEL Damage Equivalent Load

EOP Environmental Operating Points

GPR Gaussian Process Regression

$m_{B} \quad$ Whöler exponent for blade (composites)

$m_{T} \quad$ Whöler exponent for tower (welded steel)

ML Machine Learning

NMSE Normalized Mean Squared Error

RV Random Variable

$T_{i} \quad$ Turbulence intensity

$U \quad$ Mean wind speed

WT Wind Turbine

limited number of appropriately selected wind turbines in a given wind farm [34].

The work presented herein focuses on the effects induced by wakes on the Damage Equivalent Loads (DELs) on structural components of WTs. The fatigue load variation within an offshore wind farm is primarily a product of wake-induced flow disturbances [45]. The form and severity of the wake-induced turbulence and deficit depend on multiple factors, which are in this work grouped into three classes:(a) ambient conditions (wind speed, ambient turbulence, atmospheric stability, wave height, and others); (b) wind turbine operational regime (rotor thrust, rotational speed, and power set point); and (c) relative position of the wake source(s) with respect to the disturbed turbine. Recent literature has attempted to tackle the fatigue variability issue, by delivering predictive frameworks that capitalize on availability of SCADA data. The approaches in delivering such predictive models may be distinguished in terms of two main categories, namely the physicsbased and data-driven classes.

Initiating from a physics-based approach, research in Ref. [13] and in similar works $[40,44]$ exploits a surrogate approach, relying on Polynomial Chaos Expansions (PCE) and Artificial Neural networks (ANN), trained on pre-simulated load scenarios, to predict the fatigue load variation on WTs for a wind farm with arbitrary layout under wake effects. ANNs are shown to outperform PCE in terms of prediction accuracy and computational speed [43], albeit being prone to overfitting, while further require significantly more data for achieving acceptable performance. Both methods allow for obtaining analytical derivatives, which is a useful trait in optimization and sensitivity analysis. In Ref. [12] the performance of five surrogate models is assessed by comparing site-specific lifetime fatigue load predictions at 10 sites using an aeroelastic model of an individual DTU $10 \mathrm{MW}$ reference wind turbine. The compared methods include PCE, quadratic response surface, universal Kriging, importance sampling, and nearest-neighbor interpolation. The authors argue that PCE-based (and Kriging) models may sometimes have a practical advantage over ANNs, due to the "white-box" features - such as being able to track separate contributions to variance (and uncertainty). Research in Ref. [17] proposed a procedure for producing a lifetime fatigue load variation map within an offshore wind farm. Factors such as 10 -min average free wind speed, free wind direction, ambient turbulence, farm layout and wake effects, wave height, peak period, and alignment with the wind were considered. The procedure relies on direct aero-elastic simulations of the whole wind farm including wake effects using the DWM model. A similar approach was adopted in the work of Tagliatti [46]. This mapping is not directly extendable for use with continuous and long-term structural health monitoring data (SHM).

In a purely data-driven scheme, which does not take aeroelastic analyses into account, Papatheou et al. [37] focus on power prediction for the Lillgrund wind farm [2] for the purpose of condition monitoring and fault detection. They adopt both ANNs and Gaussian Processes (GPs) for producing individual and populationbased power curves. They then attempt prediction of the power produced on individual WTs based on measurements extracted from other turbines in the farm. A comparison between neural networks and GPs reveals no significant difference in terms of precision, but showcases the inherent ability of the GPs to produce probabilistic bounds. Woo et al. [49] propose a Multi-Tasks Convolutional Long Short-Term Memory Network approach to simultaneously predict the energy output and structural load from the target wind turbine, while modeling the spatio-temporal structure of the input wind flow. The work is verified on simulations from a stand-alone NREL-5MW onshore reference wind turbine. The predictions are delivered in a short-term horizon, i.e., few seconds ahead. In both [37,49] wake effects are not considered. Wake is tackled in Ref. [35], where a trained Variational Autoencoder (VAE) is exploited to map the high dimensional correlated stochastic variables over the wind-farm, such as power production and wind speed, to a parametric probability distribution of much lower dimensionality, with the ultimate goal of condition monitoring.

In a method that attempts to fuse physics-based simulators with data, in what concerns the training of predictive ML models, Dimitrov et al. [14] propose to use a combination of limited SCADA based measurements and wind turbine/farm simulations. An artificial intelligence framework is trained to forecast the future performance of the wind turbine and the fatigue life consumption of its components. If SCADA measurements, such as measured power production, wind speed and rotor speed, are available, the load mapping can be realized by training a data-driven regression model using e.g. artificial neural networks (ANN). In the absence of actual loads data, an aeroelastic model of the turbine can be used to generate a synthetic data set to serve for training. A comparison of the normalized damage equivalent blade root flap moments between measurements and simulations shows a discrepancy of the order of $10 \%-15 \%$ for some of the operating wind speeds. Park \& Park [38] present an attempt to fuse data with engineering principles via a physics-induced graph neural network (PGNN) model able to estimate the power outputs of all wind turbines in any layout under any wind conditions. An engineering wake interaction model serves as a basis function, which effectively imposes physicsinduced bias for modelling the interaction among wind turbines into the network structure. To clearly understand the role of the physics-induced weight function, the authors compare the PGNN performances to a purely data-induced approach, termed datainduced GNN (DGNN). When a target turbine in a wind farm experiences more complex wake interaction, the DGNN tends to overestimate power generations. A drawback of the proposed method is the inability to produce probabilistic output.

The overview of existing literature reveals that, on the one hand, deep learning (DL) neural network based methods (Recurrent, Convolution, GraphNets, Auto-encoders, etc.) are suited to the problem at hand, but require special understanding and tuning of the network layers to reach adequate predictive results. Moreover, these often require large training datasets in order to avoid over- 
fitting. Despite significant advancements in this field, DL methods remain black-box in their very nature and comprehensive interpretable results remain difficult (for now). A number of surrogate approaches are proposed but are focused on offering an emulator of the load responses across wind turbines in a wind farm based on pre-compiled aeroelastic simulations. A lack is noted with respect to comprehensive efforts for predicting wake-induced load effects on WTs. An important goal would be to predict the loads on unmeasured wake-affected WTs via direct input loads and inflow measurements extracted from wake-free wind turbines in the same farm. This problem is particularly relevant in a realistic SHM setting for wind farms, since an efficient monitoring scheme, should involve carefully planned and, to the degree possible, sparse structural measurements.

In this work, we propose adoption of classical Gaussian process Regression (GPR) with Bayesian learning of hyper-parameters. We argue that GPR offers a number of advantages over deep learning, or some of the further surrogate modelling approaches, in that it delivers an elegant mathematical formulation and exact inference, it offers a flexible encoding of linear constraints, it has proven robust in small low dimensional input spaces and scalar univariate (non-time series) output [6,7]. Perhaps the major advantage lies in the built-in feature for uncertainty quantification that enables effective policies for data acquisition and experimental designs, including Bayesian approaches for hyperparameters optimization. On the downside, some limitations, which ought to be acknowledged include limited scalability to large data-sets and high dimensions, as well as limited expressivity and robustness to prior assumptions, especially in relation to the choice of kernels. The former consideration does not pose an issue for the analysis presented herein, as we do not deal with output time series datasets, but instead treat aggregated features (such as DELs). Furthermore, our input dimensional space is generally limited to few essential inflow and turbine response Random Variables (RVs). Regarding the second consideration on expressivity, the choice of GPR kernels is indeed a source of uncertainty. This can be tackled when kernels are introduced as a RV, as done in Ref. [3], or alternatively Kernel selection could be performed using Approximate Bayesian Computation, as done in Ref. [5].

In order to verify the proposed GP-based approach, an exploratory and therefore simplified analysis is adopted in this work, featuring an essential setup comprised of two interacting wind turbines; a first WT situated up-wind, with the second positioned directly in the wake of the first. This simple setup allows us to illustrate our findings on utilization of the proposed framework by means of easily interpretable results. Furthermore, we hypothesise that this setup is suitable for a significant number of small to medium onshore wind farms, such as for instance the layout shown in Fig. 1. The wind rose indicates a narrow band of wind direction between North-North-East and North-North-West, resulting in single meandering wake field amongst the turbines, which is the setup adopted in this paper.

The main contributions of this work pertain to i) identification of dynamic differences between up-wind and wake-affected turbines; ii) development of GPR-based framework to estimate shortterm fatigue DELs for the wake-affected wind turbine, based off loads and inflow measurements on the up-wind turbine, and iii) recommendations for a minimal set of necessary and sufficient input random variables to predict the short-term fatigue damage equivalent loads (DEL) on a wake-affected wind turbine. In appropriately accounting for inherent uncertainties, beyond inflow and fatigue related uncertainties, in our design of experiments we inject direct aerodynamic uncertainties on the lift and drag coefficients of the airfoil sections along the span of the blade, thus affecting the rotor induction and consequently the dynamic wake evolution and transport.

The remainder of this article is organized as follows. In Section 2 we describe the uncertainties and simulations setups. In section 3 we provide an interpretation of the main output from the numerical simulations especially with respect to the effects of the uncertainties (Random Variables) in relation to the short-term DEL of various structural components on the wind turbines. In section 4 we elaborate on the framework of virtual fatigue diagnostics of the wake-affected wind turbine via Gaussian Process Regression (GPR) model, and present the ensuing results in section 5. We finish with concluding discussions and outlook in section 6 .

\section{Uncertainty modeling and wake simulations setup}

In this section, we detail our uncertainty framework and the wake meandering aero-elastic simulations setup. Three categories of RVs are considered, namely: wind inflow RVs, aerodynamic RVs and fatigue RVs.

\subsection{Inflow RVs and their stochastic models}

The variation in the structural dynamic response of wind turbines is significantly dependent on the turbulent inflow wind field conditions, including the mean wind speed, turbulence, wind shear, and inflow skewness. In accounting for these influences, we introduce the following RVs in the simulations setup: Mean wind speed, $U$, turbulence intensity, $T_{i}$, wind shear, $\alpha$, and horizontal inflow skewness, $\Psi$.

The mean wind speed follows a Weibull distribution $U \sim \mathscr{W} \mathscr{B} \mathscr{L}\left(A_{U}, K_{U}\right)$, truncated to $[4-25] \mathrm{m} / \mathrm{s}$, with parameters specified as follows:

$$
\begin{gathered}
\mathbb{E}(U)=8.5, \text { where } A_{U}=\frac{2 \times \mathbb{E}(U)}{\sqrt{\pi}} \\
K_{U}=2.0
\end{gathered}
$$

The conditional dependence between the turbulence $\sigma_{U}$ and the mean wind speed $U$ is defined in the Normal Turbulence Model described in the wind turbine design standard [1]. Here, we elect to use a reference ambient turbulence intensity $I_{r e f}=.16$ (the expected value of the turbulence intensity at $15 \mathrm{~m} / \mathrm{s}$ is called $I_{\text {ref }}$ ). This dependency is given by the local statistical moments of $\sigma_{U} \sim \mathscr{L} \mathscr{N}\left(\mu_{\sigma_{U}}, \sigma_{\sigma_{U}}^{2}\right)$ as:

$\mathbb{E}\left(\sigma_{U} \mid U\right)=I_{r e f}(0.75 u+3.8)$
$\mathbb{V}\left(\sigma_{U} \mid U\right)=\left(1.4 I_{r e f}\right)^{2}$

The wind profile above ground level is expressed using the power law relationship, which defines the mean wind speed $U$ at height $Z$ above ground as a function of the mean wind speed at hub height $U_{h}$ measured at hub height $Z_{h}$ as reference:

$\frac{U}{U_{h}}=\left(\frac{Z}{Z_{h}}\right)^{\alpha}$

where $\alpha$ is a constant called the shear exponent. The conditional dependence between the wind shear exponent $\alpha \sim \mathscr{N}\left(\mu_{\alpha}, \sigma_{\alpha}^{2}\right)$ and the mean wind speed $U$ is given by Ref. [15]: 


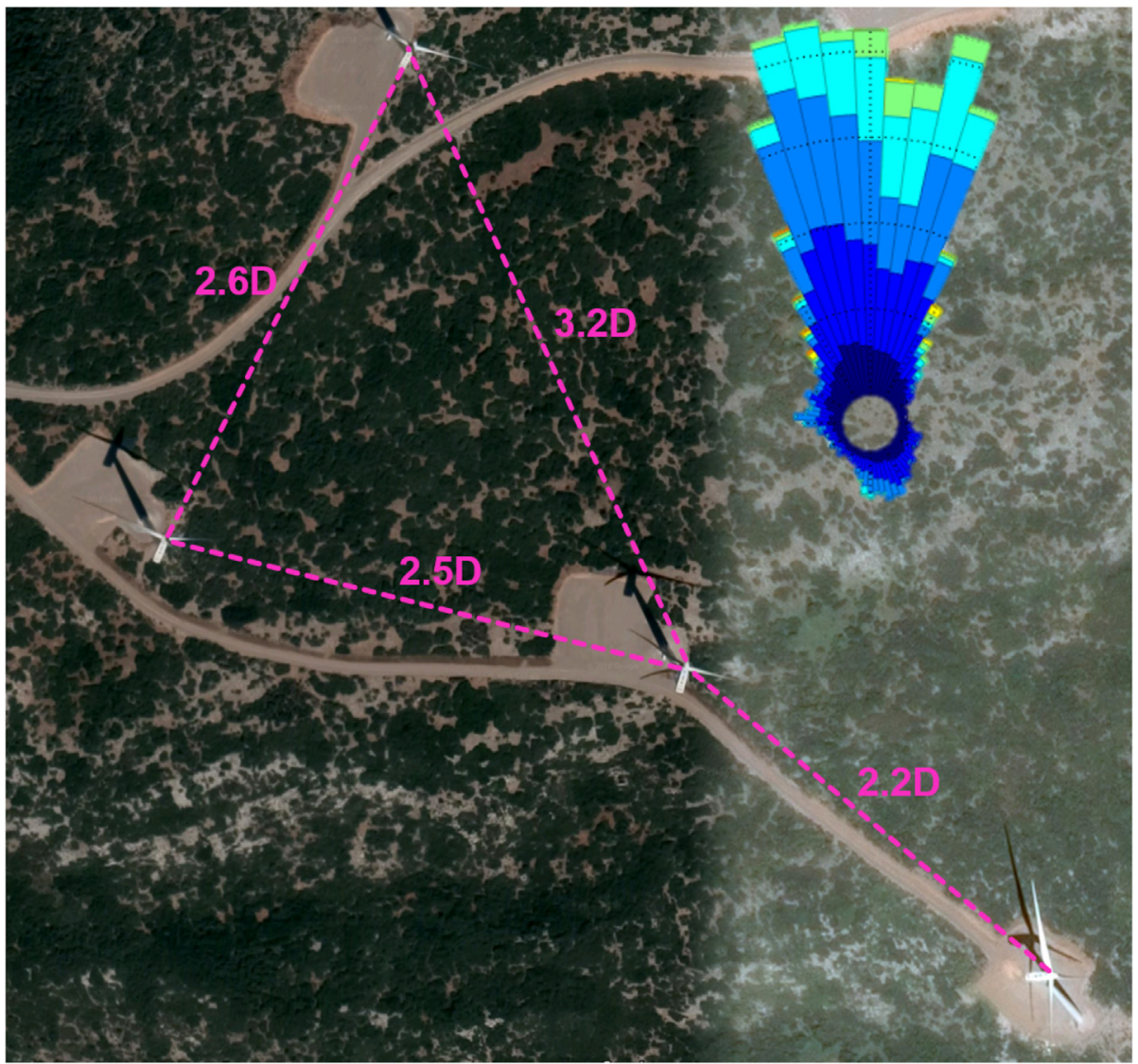

Fig. 1. Layout of an onshore wind farm in complex terrain, located in central Greece.

$$
\begin{gathered}
\mathbb{E}(\alpha \mid U)=0.088(\ln (u)-1) \\
\mathbb{V}(\alpha \mid U)=\left(\frac{1}{u}\right)^{2}
\end{gathered}
$$

We define a custom conditional dependence between the inflow horizontal skewness $\Psi$ and the mean wind speed $U$, truncated to [ $11,11] \operatorname{deg} ., \Psi \sim \mathscr{N}\left(\mu_{\Psi}, \sigma_{\Psi}^{2}\right)$ :

$$
\begin{aligned}
& \mathbb{E}(\Psi \mid U)=\ln (u)-3 \\
& \mathbb{V}(\Psi \mid U)=\left(\frac{15}{u}\right)^{2}
\end{aligned}
$$

\subsection{Aerodynamic RVs and their stochastic models}

A well know result from Blade Element Moment theory (BEM) links the aerodynamic lift $\left(C_{L}\right)$ and $\operatorname{drag}\left(C_{D}\right)$ coefficients to axial $(a)$ and tangential $\left(a^{\prime}\right)$ induction factors as follows:

$$
\begin{aligned}
\frac{a}{1-a} & =\frac{\sigma^{\prime}\left(C_{L} \cos \varphi+C_{D} \sin \varphi\right)}{4 \sin ^{2} \varphi} \\
\frac{a^{\prime}}{1-a} & =\frac{\sigma^{\prime}\left(C_{L} \sin \varphi-C_{D} \cos \varphi\right)}{4 \lambda_{r} \sin ^{2} \varphi}
\end{aligned}
$$

where $\sigma^{\prime}$ is the rotor solidity, $\Phi$ is the angle of the incoming relative wind with the rotor plane, tip speed ratio $\lambda=\frac{\omega r}{V_{0}}, \omega$ is the rotor speed, $r$ is the radial distance from the rotor center, and $V_{0}$ is the freestream wind speed. The distribution of aerodynamic axial and tangential induction over the rotor essentially governs the evolution and transport of the wake [26]. Hence, our approach to affecting aerodynamic induction is by introducing a stochastic model of the lift and drag coefficients curves in BEM. Several sources of uncertainties affect the lift and drag coefficients with direct impact on aerodynamic induction. These uncertainties are associated with assessment of airfoil characteristics in wind tunnels, uncertainties due to 3D flow correction, uncertainties stemming from surface roughness, uncertainties related to the blade geometric distortions in manufacturing and handling, uncertainties related to the blade geometric distortions when deflected under load, uncertainties due to the effects of Reynolds number, uncertainties associated with extending airfoil aerodynamic characteristics to post stall, and finally uncertainties stemming from the validation of airfoil data by field full scale measurements. It is not possible to quantify the joint distribution of all these RVs and, as a result, a simplified approach is chosen via a stochastic model, as proposed in Ref. [4]. The stochastic model consists in parameterizing the lift coefficient curve by the slope in the linear range $\frac{\partial C_{L}}{\partial \alpha}$, the point indicating the start of the trailing edge separation $\left(A O A_{T E S}\right.$, $\left.C_{L, T E S}\right)$, the point of maximum lift $\left(A o A_{\max }, C_{L, \max }\right)$ and the point where the stall recovery is initiated $\left(A o A_{S R}, C_{L, S R}\right)$. The drag coefficient is several orders of magnitude smaller than the lift coefficient for small angles of attack (below stall) and, thus, its impact is limited. Furthermore, it generally displays minor variability regardless of the airfoil type, geometry, or thickness to chord ratio. Consequently, the drag coefficient is only parameterized by the point where minimum and maximum drag coefficient occurs at $A o A=0^{\circ}$ and $A o A= \pm 90^{\circ}$, respectively. According to Ref. [4] the 
probabilistic distributions, expected values, coefficient of variations and correlation coefficients are assigned to the aforementioned parameters (for brevity, we do not repeat these here). Fig. 2 shows samples of the stochastic lift and drag coefficients curves. These perturbations result in modified $C_{L}$ and $C_{D}$ curves that maintain the primary characteristics of the original aerodynamic polars, but differ in both magnitude and feature location. Note that these synthetic aerodynamic lift and drag coefficients curves are sampled independently from the wind inflow RVs.

Furthermore, we vary the spacing between the up-wind and wake-affected WTs, as shown in Table 1.

\subsection{Sampling of the random variables}

We choose to sample the wind inflow and the aerodynamic RVs using the Sobol Quasi-Random sequences, which are designed to generate a sample that is uniformly distributed over the unit hypercube, i.e., as uniformly as possible over the multi-dimensional input space [42]. In total we sample 2048 Joint samples of the wind inflow RVs, as shown in Fig. 3. For a given spacing between the up-wind and wake-affected WTs, a sample of $U, \sigma_{U}, \alpha$ and $\Psi$, combined with a sample of stochastic $C_{L}$ and $C_{D}$, we generate a realization of an inflow turbulent wind field time series as input to the FAST-DWM aero-servo-elastic environment to simulate the corresponding aero-elastic response of the wind turbines structure.

\subsection{Fatigue RVs and their stochastic models}

In this paper, we represent fatigue using the short-term fatigue damage equivalent load (DEL) concept. The advantage of the DEL is that it reduces a long history of random loads to one number, which makes it convenient to compare various load and operating scenarios [48]. In our probabilistic calculations the exponent of the $\mathrm{S}-\mathrm{N}$ curve $m$ (Whöler exponent) is considered to be a RV. To get an impression of the influence of the Whöler exponent we compute the DEL based on a range of discrete Whöler exponents for a given material as shown in Table 2. We assume that the blades composites Whöler exponent varies between 9 and 13. We assume that the tower structural/welded steel Whöler exponent varies between 3 and 4 . The short-term fatigue damage equivalent loads (DEL) follow from the computed 10-min output time series response of the wind turbine:
$D E L=\left(\frac{1}{N_{e q}} \sum_{i} n_{i}\left(\Delta S_{i}\right)^{m}\right)^{1 / m}$

where $i$ corresponds to the number of a load cycle bin, $\Delta S_{i}$ is the cycle's load range value (including the Goodman correction), $n_{i}$ designates the number of load cycles for that bin, $m$ is the inverse of the material Whöler slope and $N_{e q}$ is the reference equivalent number of load cycles, which is calculated taking as reference frequency of $1 \mathrm{~Hz}$. The cycles in a given time series are computed using the well-known Rainflow Counting Method. For further explanations, chapter 2 from Ref. [10] can be consulted.

\subsection{Dynamic wake meandering and aero-servo-elastic simulations setup}

Our dynamic wake meandering and aero-servo-elastic simulations setup is based on the coupled DWM [33] and FAST numerical models [22]. The simulations in DWM-FAST considered two reference NREL three-bladed up-wind, horizontal-axis WT [23] with $126 \mathrm{~m}$ rotor diameter, $5 M W$ rated power and hub height of $90 \mathrm{~m}$. The rated power of $5 M W$ occurs at a wind speed of $11.4 \mathrm{~m} / \mathrm{s}$ and a rotor speed of 12.1RPM. We list some of the more important properties of the simulated wind turbine in Table 3. In the DWM setup, the two turbines are aligned as shown in Fig. 4.

FAST is a wind-turbine-specific time domain aeroelastic computer simulator that employs a combined modal and multibody dynamics formulation, adopting limited degrees of freedom (DOF). Since FAST models flexible elements using a modal representation, the reliability of this representation depends on the generation of accurate mode shapes by the engineer, which are then used as input into FAST. Large structural elements, such as blades and tower models, are characterized by properties such as stiffness and mass per unit length to represent the flexibility characteristics. FAST models the turbine using $24 \mathrm{DOF}$, including two blade-flap modes and one blade-edge mode per blade, two fore-aft and two side-toside tower bending modes, nacelle yaw, the generator azimuth angle and the compliance in the drive train between the generator and hub/rotor. The aerodynamic model is based on the Unsteady Blade Element Momentum theory, including skew inflow, dynamic stall and generalized dynamic wake [11]. The Blade aerodynamic profiles' properties are provided as a-priori input and are used as lookup tables or for interpolation. The stochastic input wind field uses the Kaimal turbulence model [24]. Aeroelastic simulations of

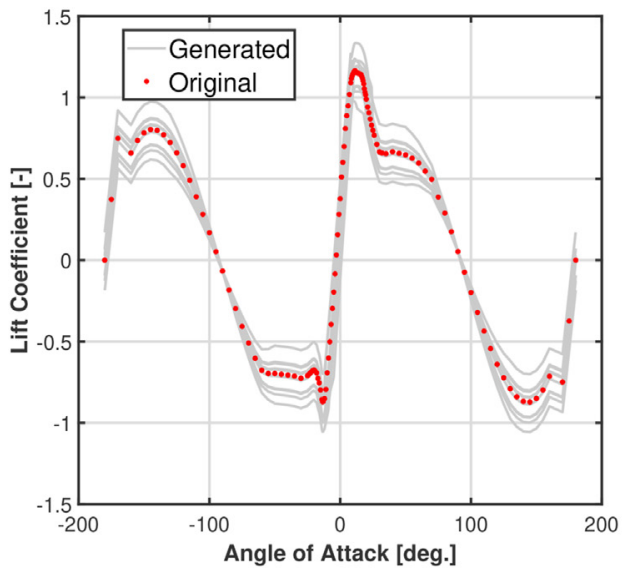

(a) $C_{L}$

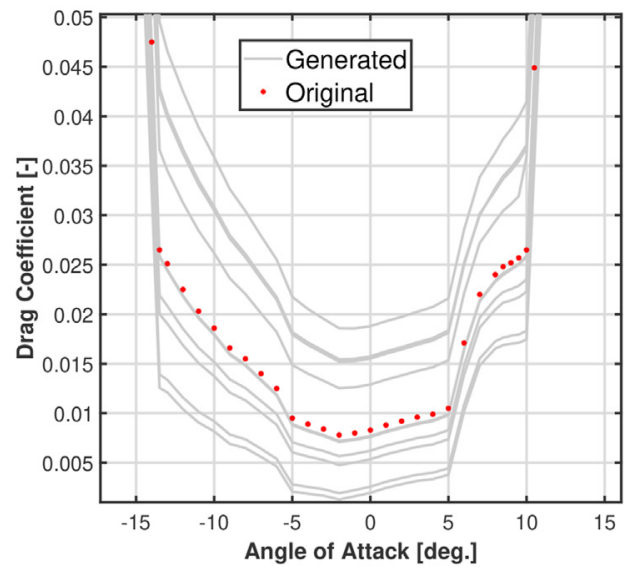

(b) $C_{D}$

Fig. 2. Samples of the stochastic lift and drag coefficients $C_{L}$ and $C_{D}$ curves of airfoil NACA 64-618. 
Table 1

Spacing between wind turbines.

\begin{tabular}{|c|c|c|c|}
\hline Random variable & Description & Probability Distribution & Parameters \\
\hline $\mathscr{D}$ & Spacing in multiples of rotor diameters between turbines & Discrete & $\mathscr{D}=[3,5,8,11]$ \\
\hline
\end{tabular}

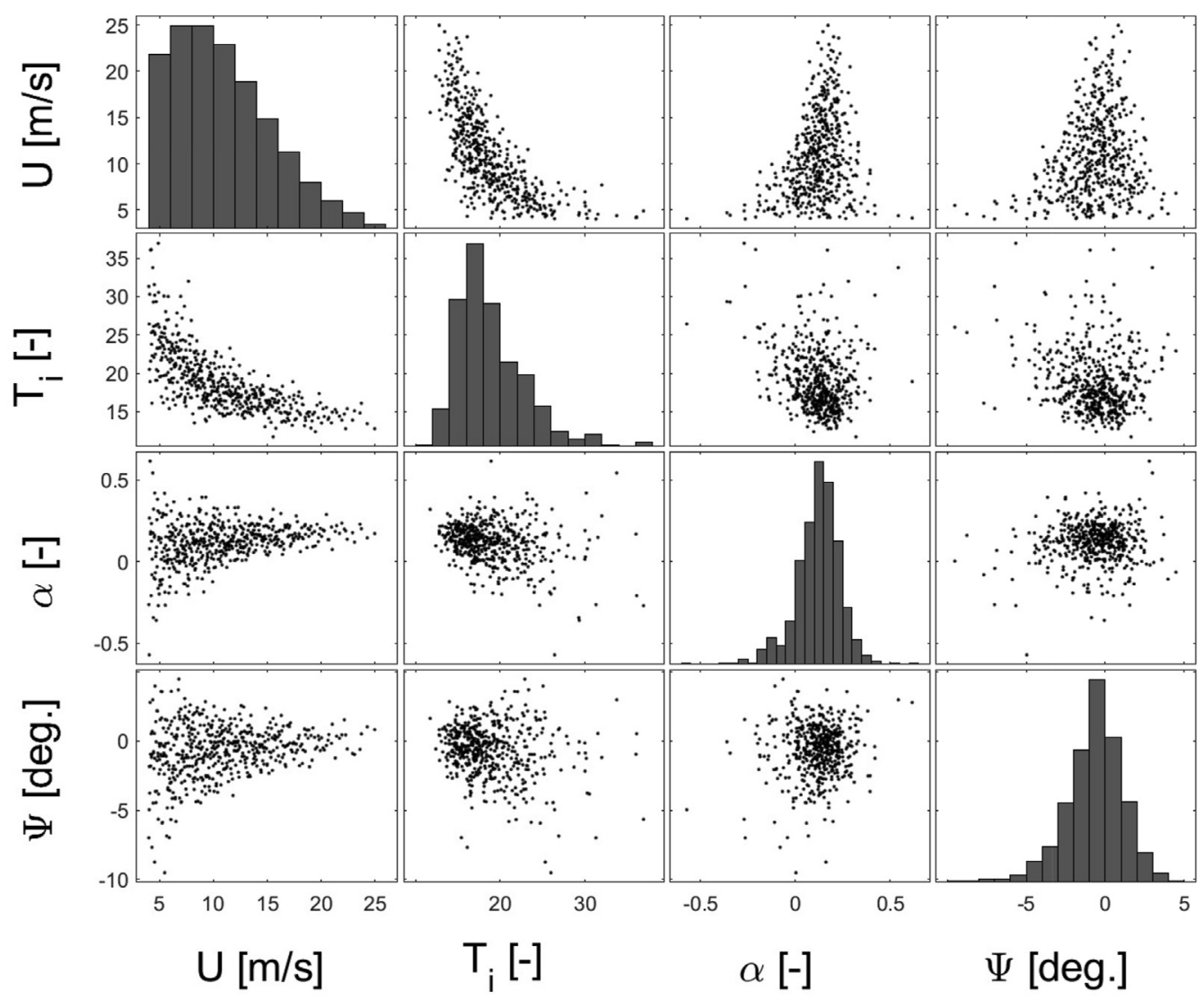

Fig. 3. Samples from the joint wind inflow random variables.

Table 2

Fatigue related random variables.

\begin{tabular}{|c|c|c|c|}
\hline Random variable & Description & Probability Distribution & Parameters \\
\hline$m_{T}$ & Whöler exponent for tower (welded steel) & Discrete & $m_{T}=[3,4]$ \\
\hline$m_{B}$ & Whöler exponent for blades (composites) & Discrete & $m_{B}=[9,10,11,12,13]$ \\
\hline
\end{tabular}

Table 3

Properties of the NREL 5-MW reference wind turbine.

\begin{tabular}{ll}
\hline Number of blades & 3 \\
\hline Rotor diameter & $126 \mathrm{~m}$ \\
Hub height & $90 \mathrm{~m}$ \\
Rated power & $5 \mathrm{MW}$ \\
Cut-in wind speed & $3 \mathrm{~m} / \mathrm{s}$ \\
Cut-out wind speed & $25 \mathrm{~m} / \mathrm{s}$ \\
Control & Variable Speed, Collective Pitch \\
Variable speed & from cut-in to cut-out wind speed \\
Variable pitch & from cut-in to cut-out wind speed \\
Rated wind speed & $11.4 \mathrm{~m} / \mathrm{s}$ \\
Cut-in and rated RPM & $6.9-12.1 R P M$ \\
\hline
\end{tabular}

WTs are stochastic largely due to the stochastic nature of the input wind field; It is thus a common practice to generate a significant number of stochastic simulations for various operating and environmental conditions in order to cover variability on aeroelastic fatigue and extreme load analysis. Wind turbines located in wind farms experience a wind field that is modified compared to the undisturbed ambient wind field. A wake is characterized by a decrease in the mean wind speed and increase in wind speed fluctuations (turbulence) behind a turbine. The downstream transport of a wake follows a stochastic pattern known as wake meandering (oscillations). It appears as an intermittent phenomenon, where winds at down-wind positions may be undisturbed for part of the time, but interrupted by episodes of intense turbulence and reduced mean speed as the wake hits the observation point [30]. Thus, a correct wind turbine load prediction requires the inclusion of the downstream evolution of wake deficit, the increased small-scale wake turbulence and the wake meandering. In this paper we choose to use the DWM wake model coupled to FAST following the NREL implementation. This coupling is well documented in Ref. [20]. The dynamic wake meander model coupled 


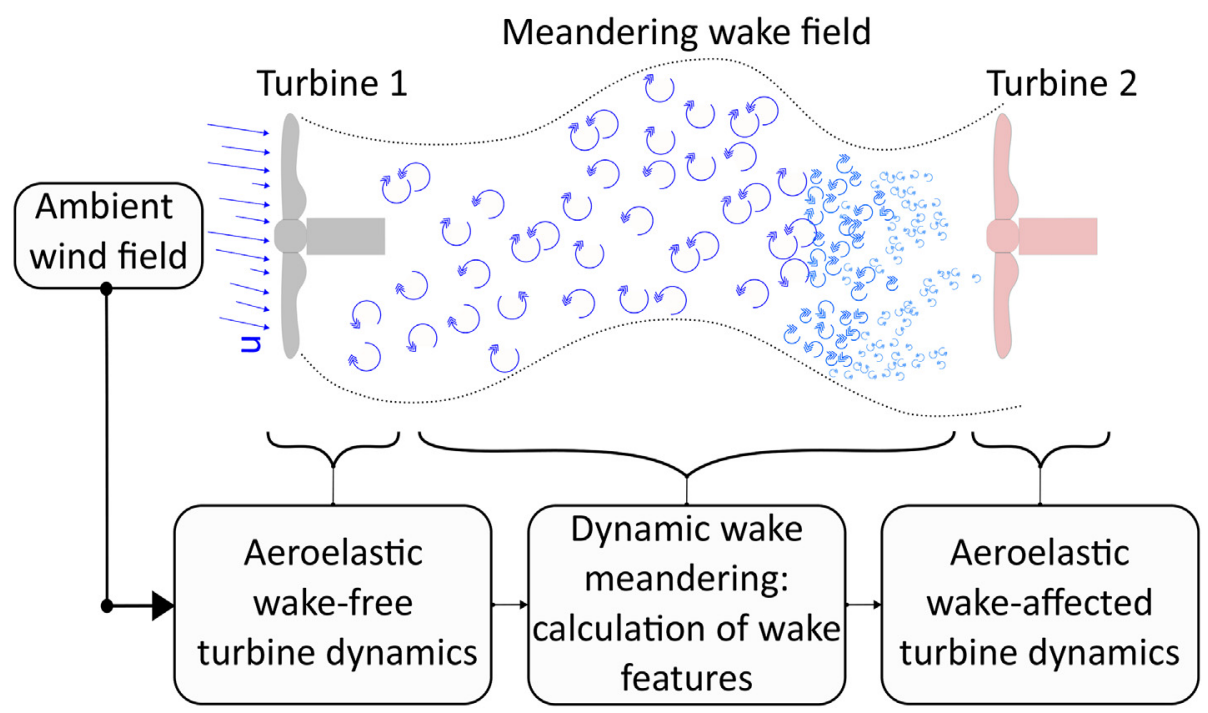

Fig. 4. Schematic of up-wind and wake-affected wind turbines with a single meandering wake field.

into FAST is used to model the up-wind (Turbine 1 in Fig. 4) wind turbine's wake effect on the structural dynamics of the down-wind wake-affected turbine (Turbine 2 in Fig. 4). While FAST is simulating an up-wind turbine, DWM calculates the wake deficit velocity, the meandered wake center positions with respect to time, and the added turbulence intensity due to the presence of the wake mixing. While a down-wind wake-affected turbine is being simulated in FAST, the inflow wind to this wake-affected turbine is modified based on the wake modelling results of its up-wind turbines. Thus, the effect of the wakes can be reflected on the wakeaffected turbine according to its immediate wake [20]. It should be noted that the wake-induced load effects are obtained under the assumptions underlying the DWM model, i. e, that the wake deficit behaves as a passive tracer following the transverse wind fluctuations and that the ambient turbulence causing the meandering can be described by a Gaussian random turbulence model, such as the Mann model. Consequently, the FAST and DWM models might suffer from model-form deficiencies and lack of inclusion of some physics, which should not distract from the main objective and thrust of this work. Our simulations setup is limited to only two turbines, with flow down a row. We would expect to see more differences in larger wind farms because of blockage and deep array effects.

\subsection{Retained sensorial output of aero-servo-elastic simulations}

Out of the hundreds of sensorial output available from our simulations, we elect to retain only four for the sake of interpretability of the results and brevity of the publication, as shown in Table 4 . These include the blade root and the tower base bending moments. Our presumption is to retain the sensorial output of aero-servo-elastic simulations that could, in the real-world, be directly deployed with little technical and economic cost if not

Table 4

Retained sensorial output of aero-servo-elastic simulations.

\begin{tabular}{ll}
\hline Sensor & Description \\
\hline TwrBsMyt & Tower base fore-aft bending moment \\
TwrBsMxt & Tower base side-side bending moment \\
RootMyb1 & Blade 1 root flapwise bending moment \\
RootMxb1 & Blade 1 root edgewise bending moment \\
\hline
\end{tabular}

already available in today's structural health monitoring systems on WTs in the field using, for instance, fiber-based strain sensing.

\section{Interpreting the simulations output}

In this section we attempt to provide an interpretation of the numerical simulations, specifically with respect to the elementary effects of the inflow RVs on the short-term DEL of the blades and tower base in relation to the wake formation and transport. A qualitative analysis is here offered with the purpose of informing the subsequent GPR and sensitivity analysis.

\subsection{Effect of mean wind speed $U$ and turbulence $\sigma_{U}$}

In Fig. 5 the up-wind wind turbine is operating below rated power, which corresponds to a high thrust coefficient and high induction, thus leading into a significant wake deficit (lower wind speed in the wake) and an increase in turbulence. When the spacing is set to $3-5 D$ the tower base fore-aft DEL of the wakeaffected wind turbine is higher compared to DEL of the up-wind wind turbine. The high thrust coefficient and high induction from the up-wind wind turbine lead in reduction of the mean wind speed and an increase in the turbulence intensity of the transported wake towards the down-wind turbine. A wake-affected wind turbine experiences increased turbulence on the basis of two main contributions. Firstly, small-scale turbulence due the breakdown of tip vortices and turbulence generated by the shear layer in the edges of the wake and secondly from the meandering of the wake deficit relative to the position of the wake-affected rotor [26]. This increase in turbulence explains why the short-term tower base fore-aft DEL (welded steel) of the wake-affected wind turbine is higher compared to DEL of the up-wind wind turbine. This does not hold true for WT separation between $8-11 D$, where turbulence and wind speed recovers, nor does it hold true for the blade root flap moment DEL. This implies a more pronounced effect of mean wind speed and the large scale effects of wake meandering on the composite blades versus turbulence for the welded steel tower. For spacing above $8 D$ the difference in DEL for both upwind and wakeaffected wind turbine are marginal for both blades and tower structures. For wind speeds that lie above rated $>11 \mathrm{~m} / \mathrm{s}$ and for a spacing of $3-5 D$, the upwind turbine tower base fore-aft DEL start to exceed that of the wake-affected wind turbine (figures omitted 


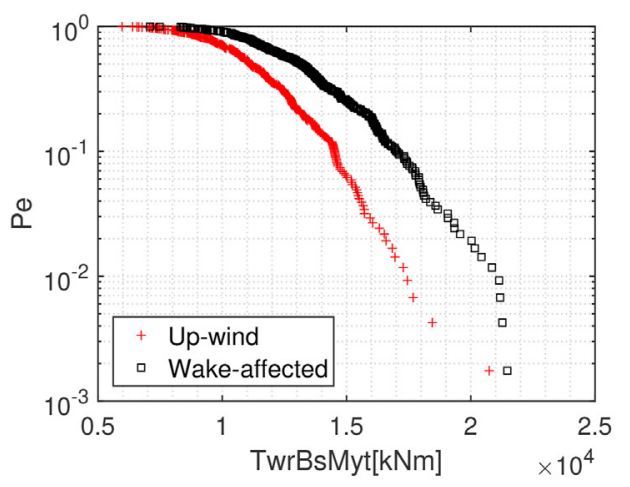

(a) Spacing $3-5 D$

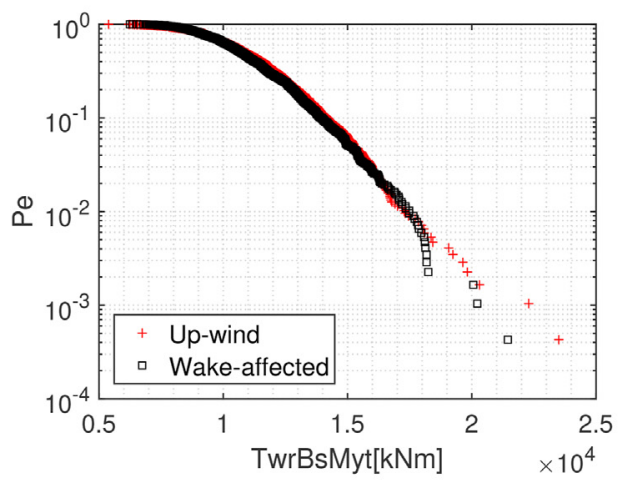

(c) Spacing $8-11 D$

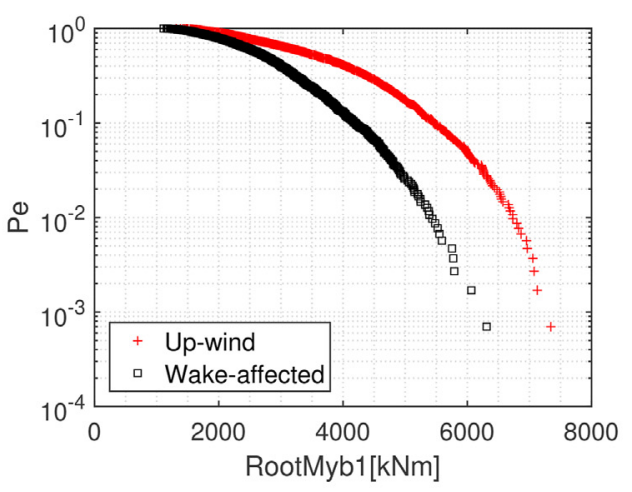

(b) Spacing $3-5 D$

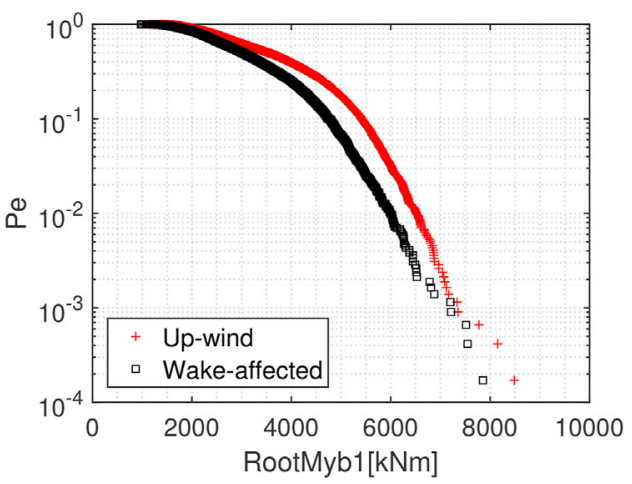

(d) Spacing $8-11 D$

Fig. 5. Exceedence probabilities for tower base fore-aft bending moment DEL and blade root flapwise bending moment DEL conditional on $u \in[3-10] m / s$.

for brevity). This implies that the small scale mixing in the wake dominates the large scale effects of wake meandering, with the mean wind speed rendered the main driver of DEL. This may be attributed to less efficient transport of the wake meandering, as a result of lower thrust coefficient and induction on the rotor at higher wind speeds. It is further noted that for increasing mean wind speed, the gap in the blade root flapwise DEL reduces gradually between the upwind and wake affected wind turbine (figures omitted for brevity).

\subsection{Effect of wind shear, $\alpha$}

Wind shear induces a periodic higher induction effect on one part of the rotor, which results in loss of symmetry for the wake deficit. This implies that elevated wind shear reduces the overall efficiency of the rotor, while creating a less severe wake in the process. This is particularly true for wind speeds above rated. This effect is well captured in Fig. 6 (c). The data is filtered to $u \in$ $[15-25] \mathrm{m} / \mathrm{s}$ above rated wind speed, where rotor induction is low to start with. The shear exponent is varied in ranges corresponding to $\alpha \in[0.05-0.11], \alpha \in[0.13-0.18]$ and $\alpha \in[0.2-0.31]$. When the shear exponent is low and in the narrow range $\alpha \in[0.05-0.11]$ the blade root flapwise bending moment DEL of the wake-affected wind turbine is shown to exceed the DEL of the up-wind wind turbine. When the shear exponent increases, the up-wind turbine exhibits higher DEL with respect to the wake-affected wind turbine. For wind speeds below rated, i.e., those corresponding to high thrust and high induction, as shown in Fig. 6a, inefficiencies due to wind shear start to appear in the tail of the exceedence probabilities (corresponding to $U \approx 10 \mathrm{~m} / \mathrm{s}$ ) for the blade root flapwise bending moment DEL of the wake-affected WT, with a reversal resulting in higher loads for $\alpha \in[0.05-0.11]$ compared to higher shear exponent ranges.

\subsection{Effect of horizontal inflow skewness $\Psi$}

In Fig. 7a the up-wind wind turbine is operating below rated power, resulting in significant wake deficit, i.e., lower wind speed in the wake, and increase in turbulence. When the spacing amounts to $3-5 D$ and the horizontal inflow skewness is negative $\Psi \in[-$ $10-1]$, the tower base side-side bending moment DEL of the wake-affected WT is higher compared to DEL of the up-wind WT. However, this difference in Fig. $7 \mathrm{~b}$ vanishes once the horizontal inflow skewness becomes positive $\Psi \in\left[\begin{array}{ll}1 & 10\end{array}\right]$. A similar effect of the horizontal inflow skewness is also observed on the blade root edgewise bending moment DEL as shown in Fig. $7 \mathrm{c}$ and $\mathrm{d}$.

\subsection{Recommendations}

This work aims to establish a data-driven model to predict the loads in the wake-affected WT, relying on loads measurements extracted from adjacent WTs, operating on availability of sparse structural measurements across the farm. From a structural health monitoring and life cycle assessment point of view, the following guidelines/recommendations can be suggested for inflow RVs:

- We recommend acquiring wind inflow turbulence data with high accuracy and precision, primarily for wind speeds below rated, when the aim lies in yielding a confident predictor/surrogate of the tower base fore-aft DEL loads of the wake-affected wind turbine.

- We recommend acquiring wind inflow shear data with high accuracy and precision, primarily for wind speeds corresponding to maximum thrust (i.e. $U \approx 10 \mathrm{~m} / \mathrm{s}$ ) and above rated wind 


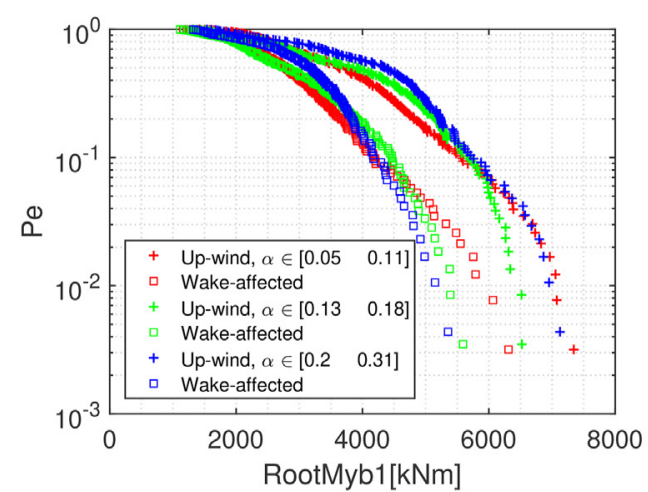

(a) $U \in[3-10] \mathrm{m} / \mathrm{s}$

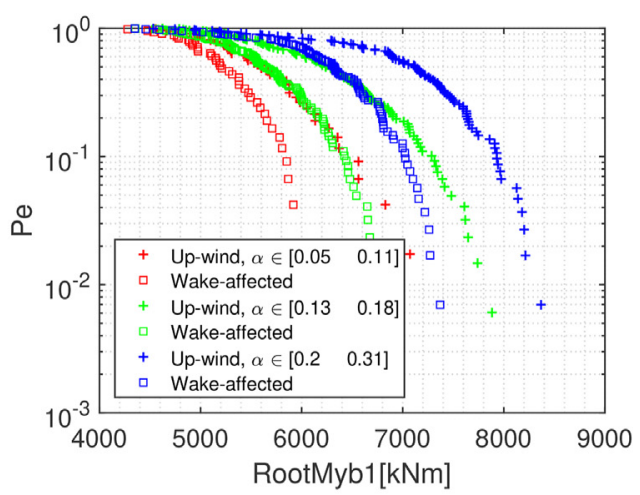

(b) $U \in[11-14] \mathrm{m} / \mathrm{s}$

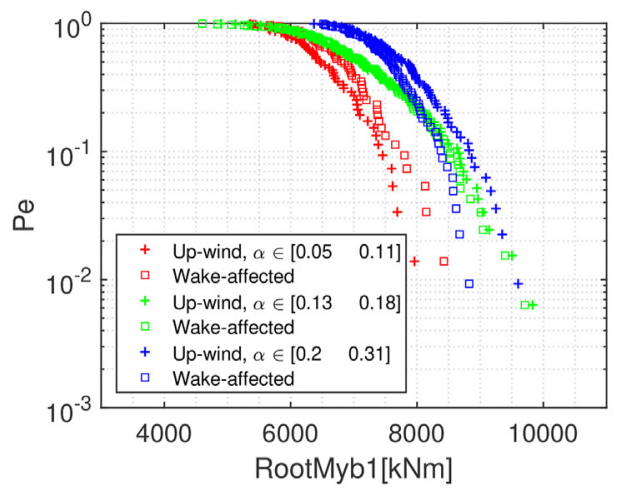

(c) $U \in[15-25] \mathrm{m} / \mathrm{s}$

Fig. 6. Exceedence probabilities for blade root flapwise bending moment DEL conditional on increasing shear exponent ranges, and spacing 3 - $5 D$.

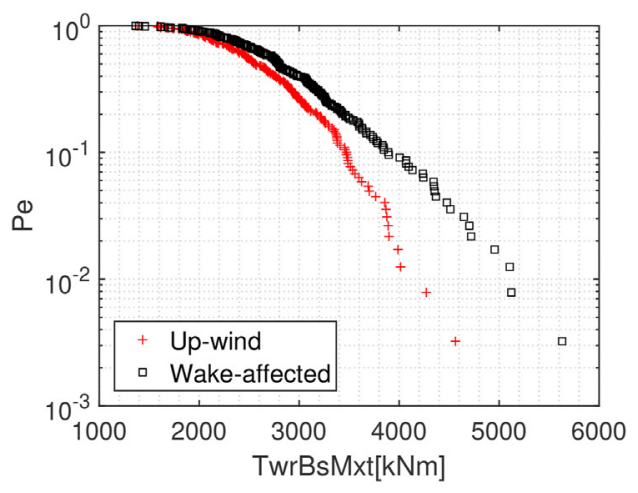

(a) $\Psi \in\left[\begin{array}{ll}-10 & -1\end{array}\right]$

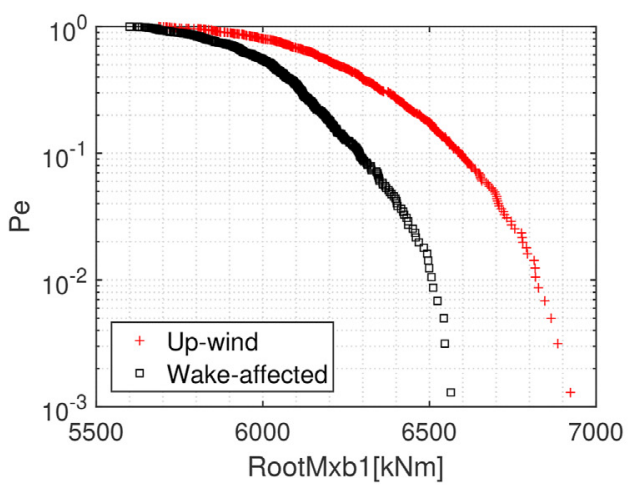

(c) $\Psi \in\left[\begin{array}{ll}-10 & -1\end{array}\right]$

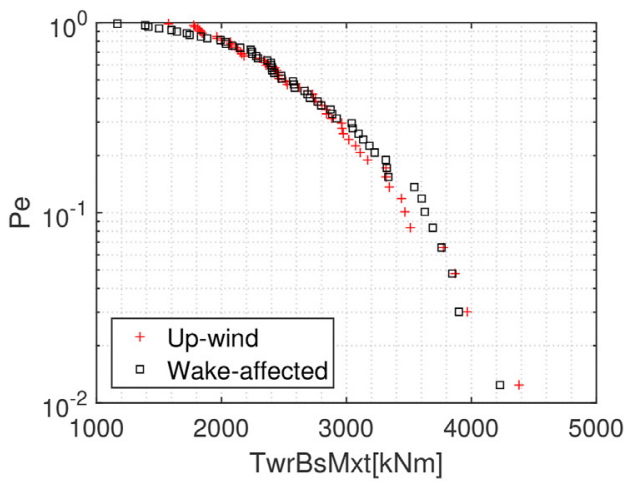

(b) $\Psi \in\left[\begin{array}{ll}1 & 10\end{array}\right]$

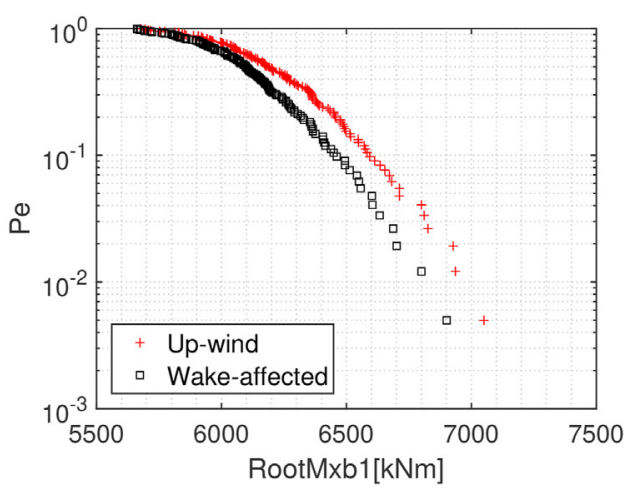

(d) $\Psi \in\left[\begin{array}{ll}1 & 10\end{array}\right]$

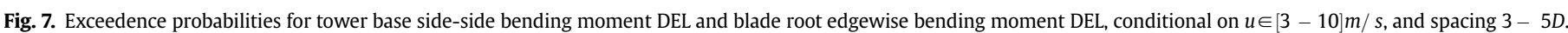


speed (i.e. $U \sim>14 \mathrm{~m} / \mathrm{s}$ ), when the aim lies in yielding a confident predictor/surrogate of blade flap DEL loads of the wake-affected wind turbine.

- We recommend acquiring horizontal inflow skewness data with high accuracy and precision, primarily for wind speeds below maximum thrust (i.e. $U \sim<10 \mathrm{~m} / \mathrm{s}$ ), when the aim lies in yielding a confident predictor/surrogate of the tower base sideside and blade root edgewise DEL loads of the wake-affected wind turbine.

\section{Methodological framework}

This section describes the generalities of the GPR method that is later used for prediction of DELs. Whilst to some extent the presented methodology follows the classical GPR framework, here we introduce a Bayesian hyperparameter identification method aided by the Metropolis-Hastings algorithm to capture the GPR modeling uncertainty. In addition, we postulate an approach based on the GPR hyperparameters to assess the sensitivity of the regressed variable to individual inputs. Finally, we propose a method based on the Kullback-Leibler divergence to compare between the DEL response surfaces observed in up-wind and wake-affected WTs using the obtained GPRs as surrogates.

\subsection{Gaussian Processes}

Consider the function $f(\boldsymbol{x}) \in \mathbb{R}$ of the input vector $\boldsymbol{x} \in \mathbb{R}^{n}$. The function $f(\cdot)$ is referred to as a Gaussian Process (GP) if its value, when sampled on a finite number of inputs $\boldsymbol{X}=\left[\begin{array}{llll}\boldsymbol{x}_{1} & \boldsymbol{x}_{2} & \cdots & \boldsymbol{x}_{N}\end{array}\right]$, follows the multivariate normal distribution $\mathscr{N}(\boldsymbol{\mu}(\boldsymbol{X}), \boldsymbol{K}(\boldsymbol{X}, \boldsymbol{X}))$, with mean $\boldsymbol{\mu}(\boldsymbol{X})=\mathrm{E}\{\boldsymbol{f}(\boldsymbol{X})\}$ and covariance $\boldsymbol{K}(\boldsymbol{X}, \boldsymbol{X})=\mathrm{E}\{(\boldsymbol{f}(\boldsymbol{X})-$ $\left.\boldsymbol{\mu}(\boldsymbol{X})) \cdot(\boldsymbol{f}(\boldsymbol{X})-\boldsymbol{\mu}(\boldsymbol{X}))^{\top}\right\}$, where $\boldsymbol{f}(\boldsymbol{X})=\left[\begin{array}{llll}f\left(\boldsymbol{x}_{1}\right) & f\left(\boldsymbol{x}_{2}\right) & \cdots & f\left(\boldsymbol{x}_{N}\right)\end{array}\right]^{T}$ [39, Sec. 2.2]. In turn, the mean and covariance are of the form:

$\boldsymbol{\mu}(\boldsymbol{X})=\left[\begin{array}{c}\mu\left(\boldsymbol{x}_{1}\right) \\ \mu\left(\boldsymbol{x}_{2}\right) \\ \vdots \\ \mu\left(\boldsymbol{x}_{N}\right)\end{array}\right] \boldsymbol{K}(\boldsymbol{X}, \boldsymbol{X})=\left[\begin{array}{cccc}k\left(\boldsymbol{x}_{1}, \boldsymbol{x}_{1}\right) & k\left(\boldsymbol{x}_{1}, \boldsymbol{x}_{2}\right) & \cdots & k\left(\boldsymbol{x}_{1}, \boldsymbol{x}_{N}\right) \\ k\left(\boldsymbol{x}_{2}, \boldsymbol{x}_{1}\right) & k\left(\boldsymbol{x}_{2}, \boldsymbol{x}_{2}\right) & \cdots & k\left(\boldsymbol{x}_{2}, \boldsymbol{x}_{N}\right) \\ \vdots & \vdots & \ddots & \vdots \\ k\left(\boldsymbol{x}_{N}, \boldsymbol{x}_{1}\right) & k\left(\boldsymbol{x}_{N}, \boldsymbol{x}_{2}\right) & \cdots & k\left(\boldsymbol{x}_{N}, \boldsymbol{x}_{N}\right)\end{array}\right]$

where $k\left(\boldsymbol{x}_{i}, \boldsymbol{x}_{j}\right)$ is the respective covariance function, a symmetric positive definite function which measures the similarity between the pair of input RVs $\boldsymbol{x}_{i}$ and $\boldsymbol{x}_{j}$. The GP is determined by the mean and covariance functions. Hereafter, the mean function is assumed as zero, while the covariance kernel is selected as the squared exponential, which is defined as follows [39, pp. 83-84]:

$k\left(\boldsymbol{x}, \boldsymbol{x}^{\prime}\right)=\sigma_{f}^{2} \cdot \exp \left(-\frac{1}{2} \sum_{i=1}^{n} \ell_{i}^{2} \cdot\left(x_{i}-x_{i}^{\prime}\right)^{2}\right)$

where $\sigma_{f}^{2}:=k(\boldsymbol{x}, \boldsymbol{x})$ is the function variance, and $\ell_{i}^{2}, i=1, \ldots, n$ are scaling factors for each one of the input dimensions. The scaling factors determine the smoothness of the function on the respective input dimension: a very large value indicates large differences between adjacent points, thus leading to non-smooth behavior; a very small value indicates significant similarity between remote points, and suggests that there are no significant variations on the signal. As will be explained later, these values are adjusted to the observed data, while the obtained scaling factors can be used to determine the influence of the input dimensions on the function outcomes.

\subsection{Gaussian Process regression}

In a Gaussian Process Regression (GPR), the covariance kernel is utilized to associate the function values observed on a set of training points, $\boldsymbol{X}=\left[\begin{array}{llll}\boldsymbol{x}_{1} & \boldsymbol{x}_{2} & \cdots & \boldsymbol{x}_{N}\end{array}\right]$, to a test input vector $\boldsymbol{x}_{*}$, and thus provide an estimate of the function value on the test point. To this end, it is first assumed that a set of noisy values of the function are observed $y_{i}, i=1, \ldots, N$, where $y_{i}=f\left(\boldsymbol{x}_{i}\right)+w_{i}$ with $w_{i}$ a zeromean normally and independently distributed process, with variance $\sigma_{w}^{2}$. The noisy function values, grouped in the vector $\boldsymbol{y}:=\left[\begin{array}{llll}y_{1} & y_{2} & \cdots & y_{N}\end{array}\right]^{T}$, and the function value on the test input $f\left(\boldsymbol{x}_{*}\right)$ are jointly normally distributed variables, as follows [39, Sec. 2.2]:

$\left[\begin{array}{c}f\left(\boldsymbol{x}_{*}\right) \\ \boldsymbol{y}\end{array}\right] \sim \mathscr{N}\left(\left[\begin{array}{c}0 \\ 0_{N \times 1}\end{array}\right],\left[\begin{array}{cc}k\left(\boldsymbol{x}_{*}, \boldsymbol{x}_{*}\right) & \boldsymbol{k}\left(\boldsymbol{x}_{*}, \boldsymbol{X}\right) \\ \boldsymbol{k}\left(\boldsymbol{X}, \boldsymbol{x}_{*}\right) & \boldsymbol{K}(\boldsymbol{X}, \boldsymbol{X})+\sigma_{w}^{2} \boldsymbol{I}_{N}\end{array}\right]\right)$

where $\boldsymbol{k}\left(\boldsymbol{x}_{*}, \boldsymbol{X}\right)=\boldsymbol{k}^{T}\left(\boldsymbol{X}, \boldsymbol{x}_{*}\right)=\left[\begin{array}{lll}k\left(\boldsymbol{x}_{*}, \boldsymbol{x}_{1}\right) & \cdots & k\left(\boldsymbol{x}_{*}, \boldsymbol{x}_{N}\right)\end{array}\right]$ is the crosscovariance between the test and the training input vectors. Then, using the properties of the multivariate normal distribution, the distribution of the function on the test input conditioned on the training noisy function values $\boldsymbol{y}$ is also Gaussian, as follows [39, Sec. 2.2]:

$p\left(f\left(\boldsymbol{x}_{*}\right) \mid \boldsymbol{y}, \boldsymbol{X}\right)=\mathscr{N}\left(\bar{f}\left(\boldsymbol{x}_{*}\right), Q\left(\boldsymbol{x}_{*}\right)\right)$

with conditional mean $\bar{f}\left(\boldsymbol{x}_{*}\right)$ and variance $Q\left(\boldsymbol{x}_{*}\right)$, which are calculated as follows:

$\bar{f}\left(\boldsymbol{x}_{*}\right)=\boldsymbol{k}_{*} \cdot\left(\boldsymbol{K}+\sigma_{w}^{2} \mathbf{I}_{N}\right)^{-1} \boldsymbol{y}$

$Q\left(\boldsymbol{x}_{*}\right)=k_{*}-\boldsymbol{k}_{*} \cdot\left(\boldsymbol{K}+\sigma_{w}^{2} \mathbf{I}_{N}\right)^{-1} \cdot \boldsymbol{k}_{*}^{T}$

and where $k_{*}:=k\left(\boldsymbol{x}_{*}, \boldsymbol{x}_{*}\right), \boldsymbol{k}_{*}:=\boldsymbol{k}\left(\boldsymbol{x}_{*}, \boldsymbol{X}\right)$, and $\boldsymbol{K}:=\boldsymbol{K}(\boldsymbol{X}, \boldsymbol{X})$.

\subsection{Bayesian approach for adjustment of the hyperparameters of} the GPR

The performance of the GPR is defined by the kernel parameters, comprised by $\sigma_{f}^{2}, \ell_{i}^{2}$ for $i=1, \ldots, n$, and $\sigma_{w}^{2}$, which are jointly referred to as the hyperparameters $\mathscr{P}:=\left\{\sigma_{w}^{2}, \sigma_{f}^{2}, \ell_{1}^{2}, \cdots, \ell_{n}^{2}\right\}$. Often, the GPR hyperparameters are optimized via maximization of the marginal likelihood, defined as follows [39, Sec. 2.3]:

$\ln p(\boldsymbol{y} \mid \boldsymbol{X}, \mathscr{P})=-\frac{1}{2} \boldsymbol{y}^{T}\left(\boldsymbol{K}+\sigma_{w}^{2} \mathbf{I}_{N}\right)^{-1} \boldsymbol{y}-\frac{1}{2}\left|\boldsymbol{K}+\sigma_{w}^{2} \mathbf{I}_{N}\right|-\frac{N}{2} \ln 2 \pi$

This is a non-linear optimization problem, which is typically solved by gradient-based non-linear optimization methods with the help of the partial derivatives of the marginal likelihood with respect to each one of the hyperparameters. This optimization results in point estimates of the hyperparameters.

Contrariwise, Bayesian methods aim at determining a distribution for the hyperparameters given the available data, based on some original assumptions on the hyperparameter distribution. Therefore, Bayesian methods aim at calculating the hyperparameter posterior distribution [41, pp. 12-13]:

$p(\mathscr{P} \mid \boldsymbol{y}, \boldsymbol{X})=p(\boldsymbol{y} \mid \boldsymbol{X}, \mathscr{P}) \cdot p(\mathscr{P}) \cdot p^{-1}(\boldsymbol{y} \mid \boldsymbol{X})$

where $p(\mathscr{P})$ is the prior hyperparameter distribution, which 
encapsulates any a-priori knowledge of the hyperparameter distribution, and $p(\boldsymbol{y} \mid \boldsymbol{X})$ comprises the model evidence, defined as follows:

$p(\boldsymbol{y} \mid \boldsymbol{X}):=\int_{\Omega} p(\boldsymbol{y} \mid \boldsymbol{X}, \mathscr{P}) \cdot p(\mathscr{P}) \mathrm{d} \mathscr{P}$

where $\Omega$ represents the space where $\mathscr{P}$ is defined.

In the case of GPR, an analytical expression for the hyperparameter posterior is not possible, due to the non-linear interaction of the hyperparameters with the likelihood. Instead, Markov Chain Monte Carlo (MCMC) methods can be used to obtain a sample of the hyperparameter posterior [41, Ch. 6-7]. In the analysis presented below, the Metropolis-Hastings algorithm is used for this purpose. Further details on the Metropolis-Hastings sampling method can be found [41, Ch. 7].

\subsection{Sensitivity analysis based on the GPR input scaling factors}

The input scaling factors $\ell_{i}^{2}$ associated with the squared exponential kernel function defined in Eq. (9) determine the smoothness of the kernel on the respective input dimension $x_{i}$. Large positive values of $\ell_{i}^{2}$ indicate a very rough behavior of the function on the dimension $x_{i}$ [39, pp. 21-22]. This happens because the correlation between values on $x_{i}$ and $x_{i}+\Delta x$, with $\Delta x$ a small increment, drops very fast. Otherwise, values of $\ell_{i}^{2}$ close to zero indicate that the function is essentially flat on the dimension $x_{i}[39$, pp. 21-22]. In this case, the increment $\Delta x$ required to produce a significant change in the correlation needs to be very large. This property can be used as a way to evaluate the sensitivity of a function approximated by a GPR to each one of the input dimensions. This is shown below.

The squared exponential kernel in Eq. (9) can be factorized as follows:

$$
\begin{gathered}
k\left(\boldsymbol{x}, \boldsymbol{x}^{\prime}\right)=\sigma_{f}^{2} \cdot \prod_{i=1}^{n} \exp \left(-\frac{1}{2} \ell_{i}^{2} \cdot\left(x_{i}-x_{i}^{\prime}\right)^{2}\right)=\sigma_{f}^{2} \cdot \prod_{i=1}^{n} k_{i}\left(x_{i}, x_{i}^{\prime}\right) \\
k_{i}\left(x_{i}, x_{i}^{\prime}\right):=\exp \left(-\frac{1}{2} \ell_{i}^{2} \cdot\left(x_{i}-x_{i}^{\prime}\right)^{2}\right)
\end{gathered}
$$

and thus the contribution of input $x_{i}$ to the kernel can be decoupled. The value $\Delta x_{\rho}$ is here defined as the increment in $x_{i}$ required to decrease by a value $\rho$ the maximum covariance value. More precisely $\Delta x_{\rho} \in \mathbb{R}^{+}$is the value such that:

$k_{i}\left(x_{i}, x_{i}+\Delta x_{\rho}\right)=k_{i}\left(x_{i}, x_{i}\right)-\rho$

with $0<\rho \ll 1$ a small positive number. Applying the definition of $k_{i}\left(x_{i}, x_{i}^{\prime}\right)$ and using the fact that $k_{i}\left(x_{i}, x_{i}\right)=1$, then:

$\exp \left(-\frac{1}{2} \ell_{i}^{2} \cdot \Delta x_{\rho}^{2}\right)=1-\rho$

and then, solving for $\Delta x_{\rho}$, the following value is obtained:

$\Delta x_{\rho}=\ell_{i}^{-1} \cdot \sqrt{-2 \cdot \ln (1-\rho)}$

The value $\Delta x_{\rho}$ can be interpreted as the distance required to move along the $i$-th input to decrease the correlation (similarity) between the function values $f\left(x_{i}\right)$ and $f\left(x_{i}+\Delta x_{\rho}\right)$ by the value $\rho$. If the value of $\Delta x_{\rho}$ is larger than the range of the data on the $i$-th input, then the desired change in the correlation is not feasible. With $\rho$ close to zero, this result would be an indicator of flatness of the function in the direction $x_{i}$. In turn, this indicates that the function $f(\boldsymbol{x})$ is insensitive to the input $x_{i}$.

Note that although $\Delta x_{\rho}$ can just be interpreted as the inverse of $\ell_{i}$, and both variables hold the same information, $\Delta x_{\rho}$ facilitates understanding of the GPR sensitivity towards a certain input.

\subsection{Kullback-Leibler divergence for comparison of GPRs}

Consider two GPRs represented as $\mathscr{M}_{a}:=\left\{\boldsymbol{y}_{a}, \boldsymbol{X}_{a}, \mathscr{P}_{a}\right\}$ and $\mathscr{M}_{b}$ : $=\left\{\boldsymbol{y}_{b}, \boldsymbol{X}_{b}, \mathscr{P}_{b}\right\}$, with different training data and hyperparameters. Then, it is necessary to evaluate whether or not the prediction of GPRs $\mathscr{M}_{a}$ and $\mathscr{M}_{b}$ at a test point $\boldsymbol{x}_{*}$ is the same. Considering that the GPR prediction at the test point follows a Gaussian distribution, it is possible to use the Kullback-Leibler (KL) divergence to compare if both predictive distributions are the same [9, p. 57]. The KL divergence for a Gaussian distribution takes the form:

$D_{K L}\left(\boldsymbol{x}_{*} \mid \mathscr{M}_{a}, \mathscr{M}_{b}\right)=\frac{1}{2}\left(\frac{Q_{a}\left(\boldsymbol{x}_{*}\right)}{Q_{b}\left(\boldsymbol{x}_{*}\right)}+\frac{\left(\bar{f}_{a}\left(\boldsymbol{x}_{*}\right)-\bar{f}_{b}\left(\boldsymbol{x}_{*}\right)\right)^{2}}{Q_{b}\left(\boldsymbol{x}_{*}\right)}+\ln \frac{Q_{b}\left(\boldsymbol{x}_{*}\right)}{Q_{a}\left(\boldsymbol{x}_{*}\right)}-1\right)$

where $\bar{f}_{j}\left(\boldsymbol{x}_{*}\right)$ and $Q_{j}\left(\boldsymbol{x}_{*}\right)$, with $j=\{a, b\}$, are the GPR predictive mean and variance calculated with Eq. (12) for each corresponding model.

The global KL divergence of the predictions obtained with both GPRs can be obtained by integrating over the whole domain $\mathscr{X} \subseteq \mathbb{R}^{n}$, as follows:

$D_{K L}\left(\mathscr{M}_{a}, \mathscr{M}_{b}\right)=\int_{\mathscr{X}} D_{K L}\left(\boldsymbol{x} \mid \mathscr{M}_{a}, \mathscr{M}_{b}\right) \mathrm{d} \boldsymbol{x}$

while a marginalized KL divergence for input $x_{i}, i=1, \ldots, n$ can be obtained by integrating with respect to the remaining inputs, as follows:

$D_{K L}\left(x_{i} \mid \mathscr{M}_{a}, \mathscr{M}_{b}\right)=\int_{\mathscr{X}_{\sim i}} D_{K L}\left(\boldsymbol{x} \mid \mathscr{M}_{a}, \mathscr{M}_{b}\right) \mathrm{d} \boldsymbol{x}_{\sim i}$

where $\boldsymbol{x}_{\sim i}$ represents the input vector after eliminating input $x_{i}$, and $\mathscr{X}_{\sim i}$ is its respective space. Evaluation of the integrals in Eqns. (21) and (22) is not analytically tractable, and instead, numerical approximations are required. The construction of the global and marginalized KL divergences in Eqns. (21) and (22) is based on the assumption that there are no cross-correlations in the predictive distribution, or more precisely,

$\mathrm{E}\left\{\left(f\left(\boldsymbol{x}_{*_{1}}\right)-\bar{f}\left(\boldsymbol{x}_{*_{1}}\right)\right) \cdot\left(f\left(\boldsymbol{x}_{*_{2}}\right)-\bar{f}\left(\boldsymbol{x}_{*_{2}}\right)\right) \mid \boldsymbol{y}, \boldsymbol{X}\right\}=0$

for $\boldsymbol{x}_{*_{1}} \neq \boldsymbol{x}_{*_{2}}$. Although this assumption does not comply with the definition of the GP, it largely simplifies the calculation of the global and marginalized KL divergences.

\section{Results}

\subsection{Prediction and analysis of DELs from local EOPS}

In this initial analysis, GPR models are built to predict the DELs of a single WT component based on locally measured EOPs. This corresponds to the ideal case when all the WTs are fully instrumented and a complete set of wind field parameters are available on each wind turbine. The objective of this initial analysis is to determine which input variables mostly affect the DELs and to 
determine if there are significant differences between the loads in the up-wind and wake-affected WTs at different structural components.

Accordingly, in the present case the input vector $\boldsymbol{x} \in \mathbb{R}^{4}$ is made up by the $10 \mathrm{~min}$ averages of the wind speed $U:=x_{1}$, turbulence intensity $\sigma:=x_{2}$, shear exponent $\alpha:=x_{3}$, and inflow horizontal skewness $\Psi:=x_{4}$ of the respective WT. In turn, the output $y$ corresponds to the DELs calculated from the respective 10 min loads measured either in the root of one of the blades in the edgewise or flapwise directions, or in the tower base in the fore-aft or side-toside directions. For the construction of the regression, the range of the input variables is normalized within the interval $[0,1]$, while the values of the DELs are scaled down by a factor of $10^{4}$. This normalization is used to enhance the numerical stability of the models. Individual GPRs are built for the DELs obtained on the upwind and wake-affected WTs at different spacing configurations and with different Wöhler exponents.

The GPR-driven numerical analysis methodology of the DWMFAST simulations is summarized in Fig. 8. First, the GPR hyperparameters are identified based on the Bayesian optimization method facilitated by Metropolis-Hastings sampling. The obtained hyperparameter distribution is then used to perform sensitivity analysis, while expected values are employed to perform predictions used for construction of response surfaces and comparison of DELs in up-wind and wake-affected WTs.

\subsubsection{Hyperparameter identification}

The Bayesian inference approach based on the MetropolisHastings $(\mathrm{MH})$ sampling algorithm described in Section 4.3, is used to estimate of the posterior hyperparameter distribution of the GPR predictor based on the available coupled DWM-FAST simulations. To this end, 180 input-output pairs are randomly selected to calculate the GPR's marginal likelihood (Eq. (13)) within the $\mathrm{MH}$ sampling loop, while the remaining ones are used for posterior model validation. To ensure even distribution of the training samples, a sampling approach based on clustering of the complete set of inputs is performed. More precisely, an agglomerative hierarchical clustering tree based on Ward's linkage on the Euclidean distance is applied. Subsequently, 180 clusters are built based on the obtained linkage and a single input and its respective output are randomly extracted from each one of them.

Independent log-normal distributions are defined for each one of the hyperparameters. Similarly, independent log-normal distributions are selected as proposal distributions. The parameters of these distributions are summarized in Table 5.

Using the previously described set-up, a total of 10000 samples are simulated with the $\mathrm{MH}$ algorithm. Fig. 9 displays the distribution of the GPR hyperparameters based on the sample of the hyperparameter posterior drawn with the $\mathrm{MH}$ algorithm, obtained with the data from the up-wind WT tower edgewise and flapwise DELs. It is first noted that although the initial values and prior distributions are similar for all the GPR hyperparameters, the posterior distributions converge to different intervals. The distributions for the noise variance, kernel variance and the first three scale parameters $\left(\ell_{1}^{2}\right.$ : wind speed; $\ell_{2}^{2}$ : turbulence intensity; $\ell_{3}^{2}$ : shear exponent) are quite narrow, $\ell_{4}^{2}$ : horizontal inflow skewness, has wider distributions in both edgewise and flapwise DELs. The latter seems to indicate that the horizontal inflow skewness has a reduced effect in the DELs. Further analysis based on the obtained GRPs is provided in the sequel.

\subsubsection{Analysis of DELs based on the obtained GPRS}

5.1.2.1. Response surfaces. After estimation of the hyperparameter posterior, it is possible to calculate response surfaces of the DEL for any values in the input space. For instance, 1D slices displaying the DEL as a function of single input variables while the remaining ones are kept fixed can be calculated with the help of the obtained GPR. Figs. 10 and 11 display slices of the DEL in the blade -edgewise and flapwise direction- extracted from the GPR with Maximum A Posteriori (MAP) hyperparameter estimates on the up-wind and downwind WTs. On each frame, the remaining inputs are set to the sample median values, while the spacing between WTs is 11 rotor diameters, and the Wöhler exponent is $m=9$. In both cases, the GPR predictive mean is similar in both the up-wind and wakeaffected WTs, while a significant decrement is found in the DEL of the wake-affected WT at high wind speeds. On the other hand, the confidence intervals are well confined around the mid-part of the input range, while the dispersion increases towards the boundaries of the input ranges. The increased dispersion is observed due to the reduced number of points towards the boundaries.

Fig. 12 shows the GPR predictive mean of the DEL in the blade flapwise direction as a function of wind speed and turbulence intensity ( $\alpha$ and $\Psi$ set to their median values, $d=11$, and $m=9$ ). DEL estimates with variance higher than 50 times the minimum predictive variance are censored in the displayed surfaces. As observed in Fig. 11, the DEL in the blade flapwise direction increases as the wind speed and the turbulence intensity do, with the wind speed making the maximum effect. The training data points, displayed as

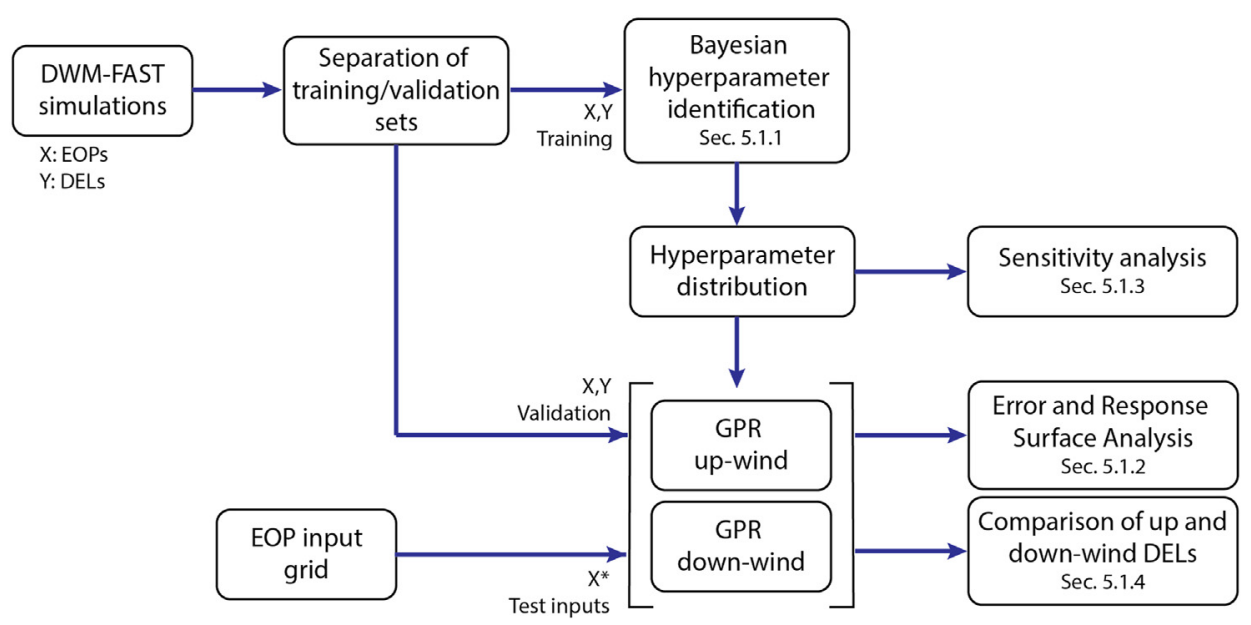

Fig. 8. Flowchart summarizing the GPR-based numerical analysis methodology of the DWM-FAST simulations. 
Table 5

Settings of the Metropolis-Hastings algorithm for sampling of the GPR hyperparameter posterior.

\begin{tabular}{llr}
\hline Hyperparameter & Prior & Proposal \\
\hline $\begin{array}{l}\text { Kernel variance } \\
\sigma_{f}^{2}\end{array}$ & $\ln \sigma_{f}^{2} \sim \mathscr{N}\left(-1,10^{2}\right)$ & $\ln \sigma_{f}^{2} \mid \ln \left(\sigma_{f}^{2}\right)\left(\sigma_{f}^{2}\right)_{-} \sim \mathscr{N}\left(\ln \left(\sigma_{f}^{2}\right)_{k-1}, 0.4\right)$ \\
$\begin{array}{l}\text { Kernel scaling } \\
\ell_{i}^{2}, i=1, \ldots, 4\end{array}$ & $\ln \ell_{i}^{2} \sim \mathscr{N}\left(0,10^{2}\right)$ & $\ln \ell_{i}^{2} \mid \ln \left(\ell_{i}^{2}\right)\left(\ell_{i}^{2}\right)_{-} \sim \mathscr{N}\left(\ln \left(\ell_{i}^{2}\right)_{-}, 0.4\right)$ \\
$\begin{array}{l}\text { Noise variance } \\
\sigma_{w}^{2}\end{array}$ & $\ln \sigma_{w}^{2} \sim \mathscr{N}\left(-1,10^{2}\right)$ & $\ln \sigma_{w}^{2} \mid \ln \left(\sigma_{w}^{2}\right)\left(\sigma_{w}^{2}\right)_{-} \sim \mathscr{N}\left(\ln \left(\sigma_{w}^{2}\right)_{-}, 0.4\right)$ \\
\hline
\end{tabular}

Number of Monte-Carlo samples $10^{4}$. The symbols $\left(\sigma_{f}^{2}\right)_{-},\left(\ell_{i}^{2}\right)_{-}$, and $\left(\sigma_{w}^{2}\right)_{-}$indicate the values of the same quantity drawn in the previous iteration of the MH sampling algorithm.
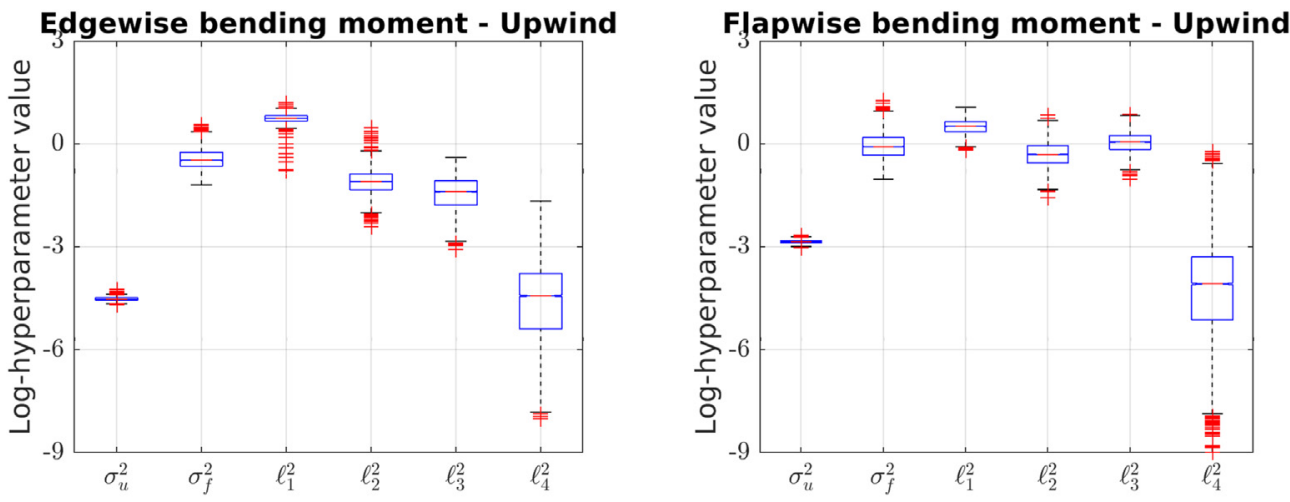

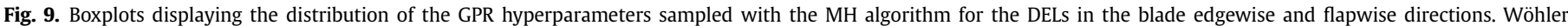
exponent: 9 .

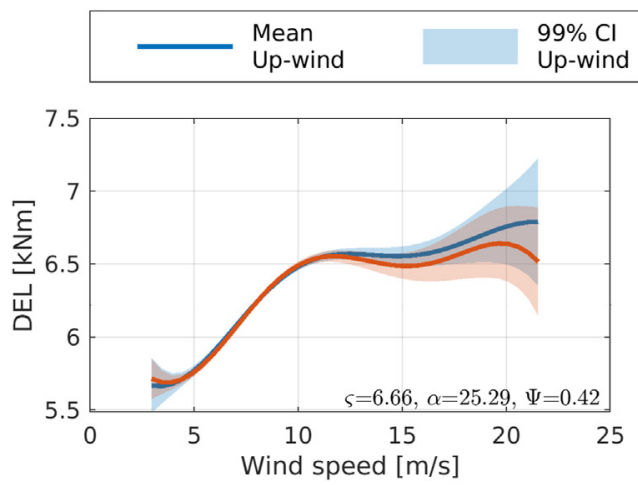

\begin{tabular}{ll} 
Mean & $99 \% \mathrm{Cl}$ \\
Down-wind & Down-wind \\
\hline
\end{tabular}
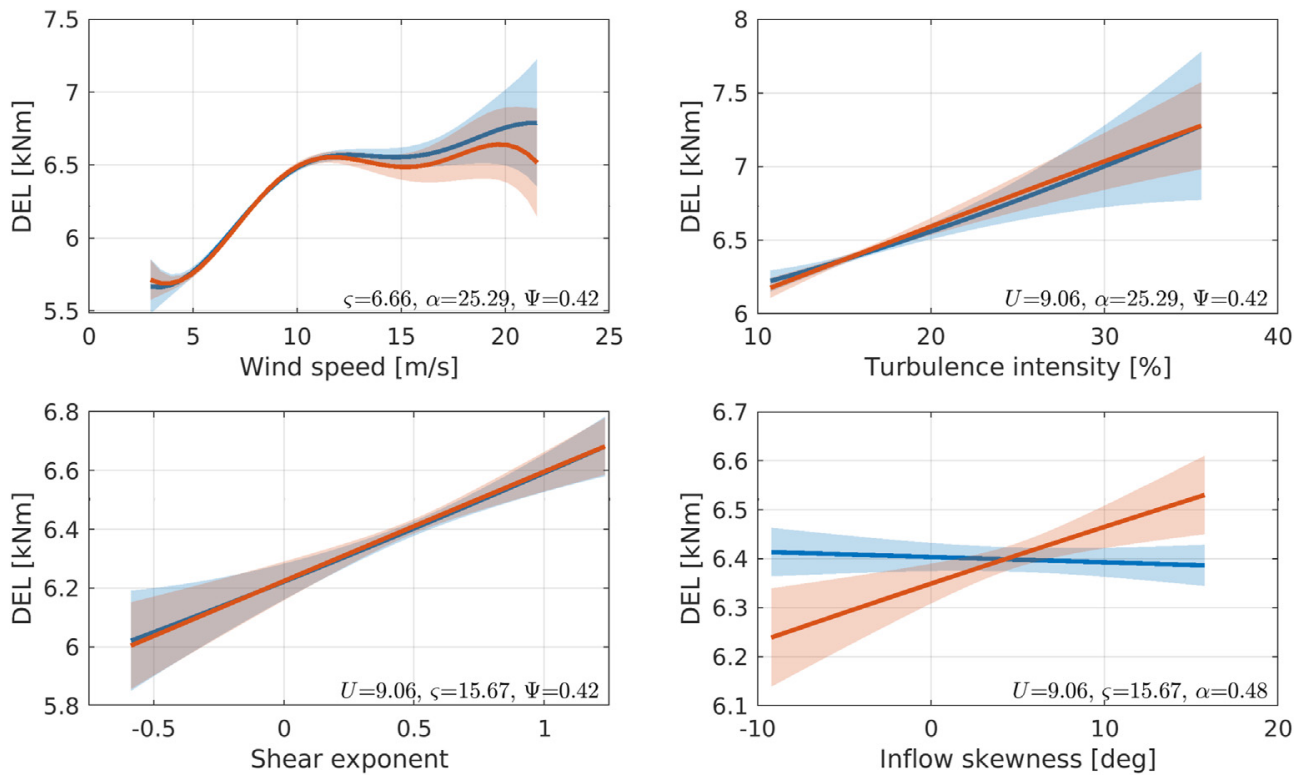

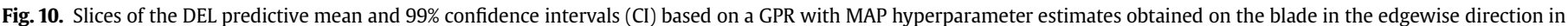
the up-wind and down-wind WTs. Other inputs are kept at their training set sample median values indicated on each frame. WT spacing: 11 diameters; Wöhler exponent: 9.

red dots in the surface, coincide with the GPR predictive mean, but also are confined to the non-censored area, which indicates that the predictive variance is well-bounded around the area spanned by the training points. In practice, DEL estimates in points outside the non-censored area can be deemed as unreliable, while at the same time have a low probability of occurrence, according to the joint distribution of $U$ and $\sigma$.

5.1.2.2. Error analysis. The predictive performance of the GPR is measured in terms of the Normalized Mean Squared Error (NMSE) 

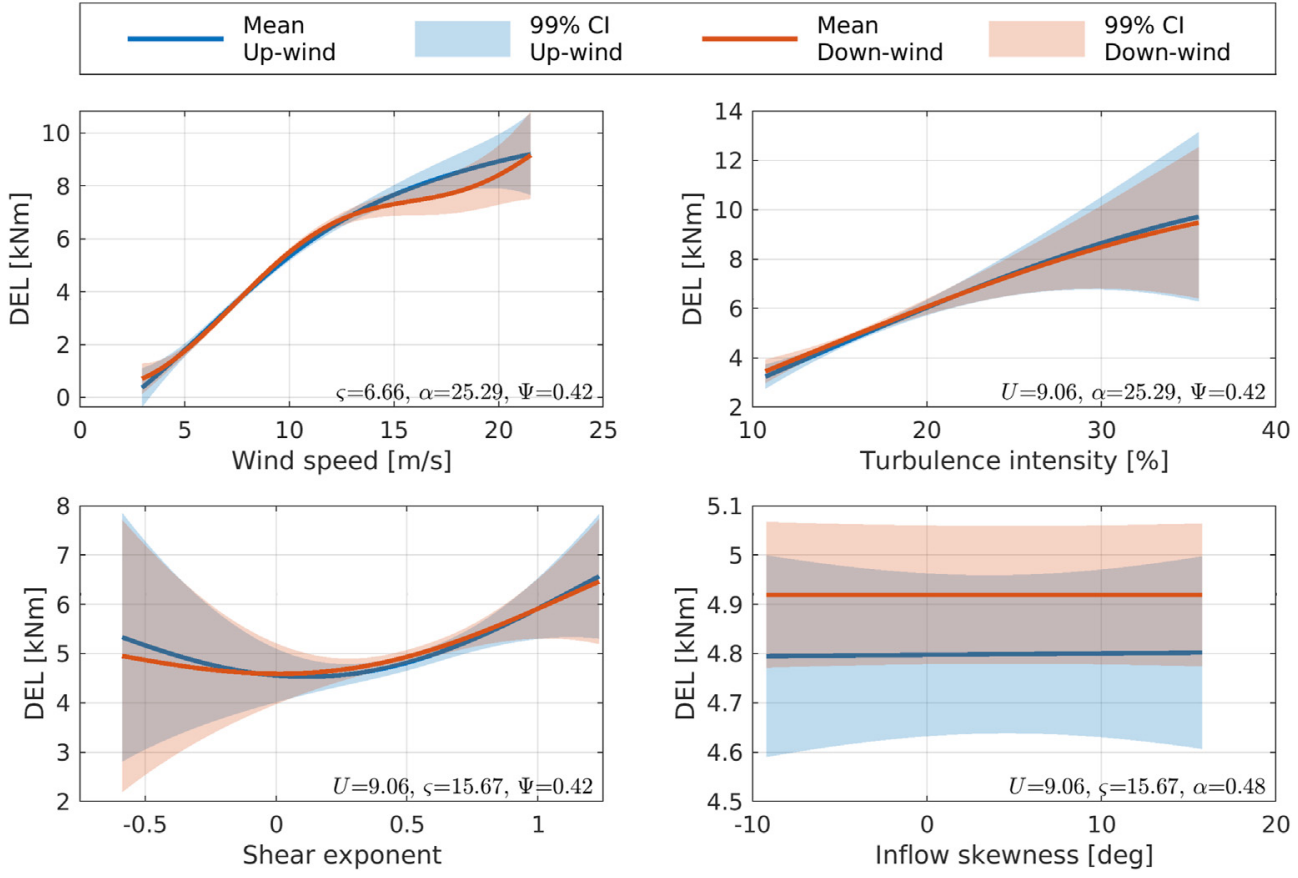

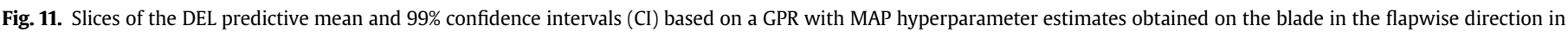
the up-wind and down-wind WTs. Other inputs are kept at their training set sample median values indicated on each frame. WT spacing: 11 diameters; Wöhler exponent: 9.
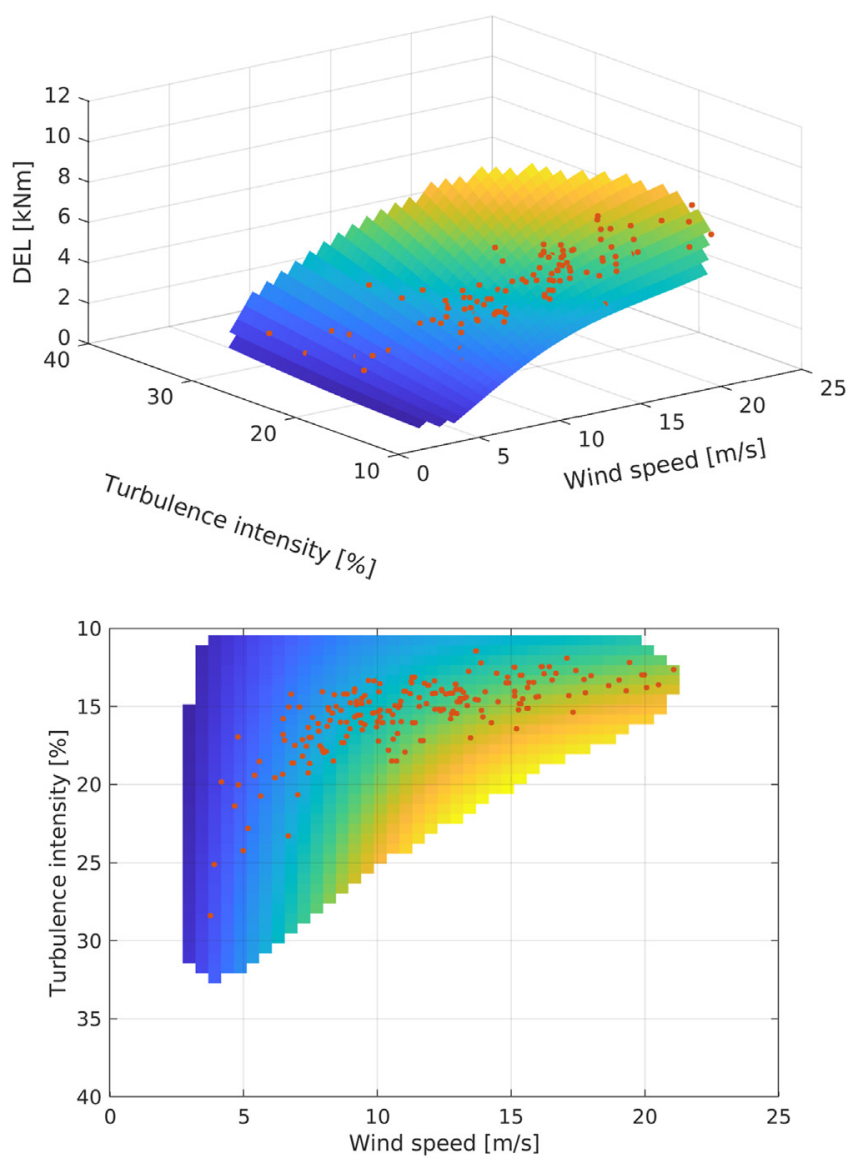

Fig. 12. Surface displaying the GPR predictive mean of the DEL in the blade flapwise direction as a function of wind speed and turbulence intensity, with the shear exponent and horizontal inflow skewness set on their median values. Wöhler exponent: 9. Red dots indicate input/output data points. calculated on the validation set. The NMSE is defined as:

$\mathrm{NMSE}=\frac{\sum_{i=1}^{N_{\text {val }}}\left(y_{i}-\bar{f}\left(\boldsymbol{x}_{i}\right)\right)^{2}}{\sum_{i=1}^{N_{\text {val }}} y_{i}^{2}}$

where $N_{v a l}$ is the number of samples in the validation set. Fig. 13 displays the NMSE obtained on the prediction of the DELs in the blade and tower on the up-wind and wake-affected WTs, with the wake-affected WT located at 11 rotor diameters. The error figures in all the cases are quite low, indicating a fair predictability in all cases, while the performance in the up-wind and wake-affected WTs is similar. In the worst case, corresponding to the tower side-to-side loads, the NMSE is under $3 \%$. On the other hand, the best case, corresponding to the blade flapwise loads, the NMSE is about two levels of magnitude lower than that in the tower side-to-side loads. For the blade loads, the NMSE appears to mildly increase with the Wöhler exponent. This tendency is followed by both up-wind and down-wind WTs.

Fig. 14 displays the NMSE as a function of the spacing between WTs for the blade and tower loads in the wake-affected WT. The overall error performance does not evidence large variations at different spacing to the values observed in Fig. 13. Moreover, the magnitude order of the NMSE is the same as that found in the previous analysis.

\subsubsection{Sensitivity analysis based on the GPR hyperparameters}

A sensitivity analysis based on the increment method, described in Section 4.4, is performed on the hyperparameter sample obtained from the GPR hyperparameter posterior with the MH sampling algorithm. Fig. 15 displays the log-increments obtained for the DELs of different components in the up-wind WT as a function of the Wöhler exponent for a correlation reduction of $\rho=0.001$. The top frame of Fig. 15 displays the log-increments obtained in the case of the loads in the blade edgewise direction. According to the results, the wind speed requires the lowest variation to perform a change in the DEL, with the turbulence intensity and shear 

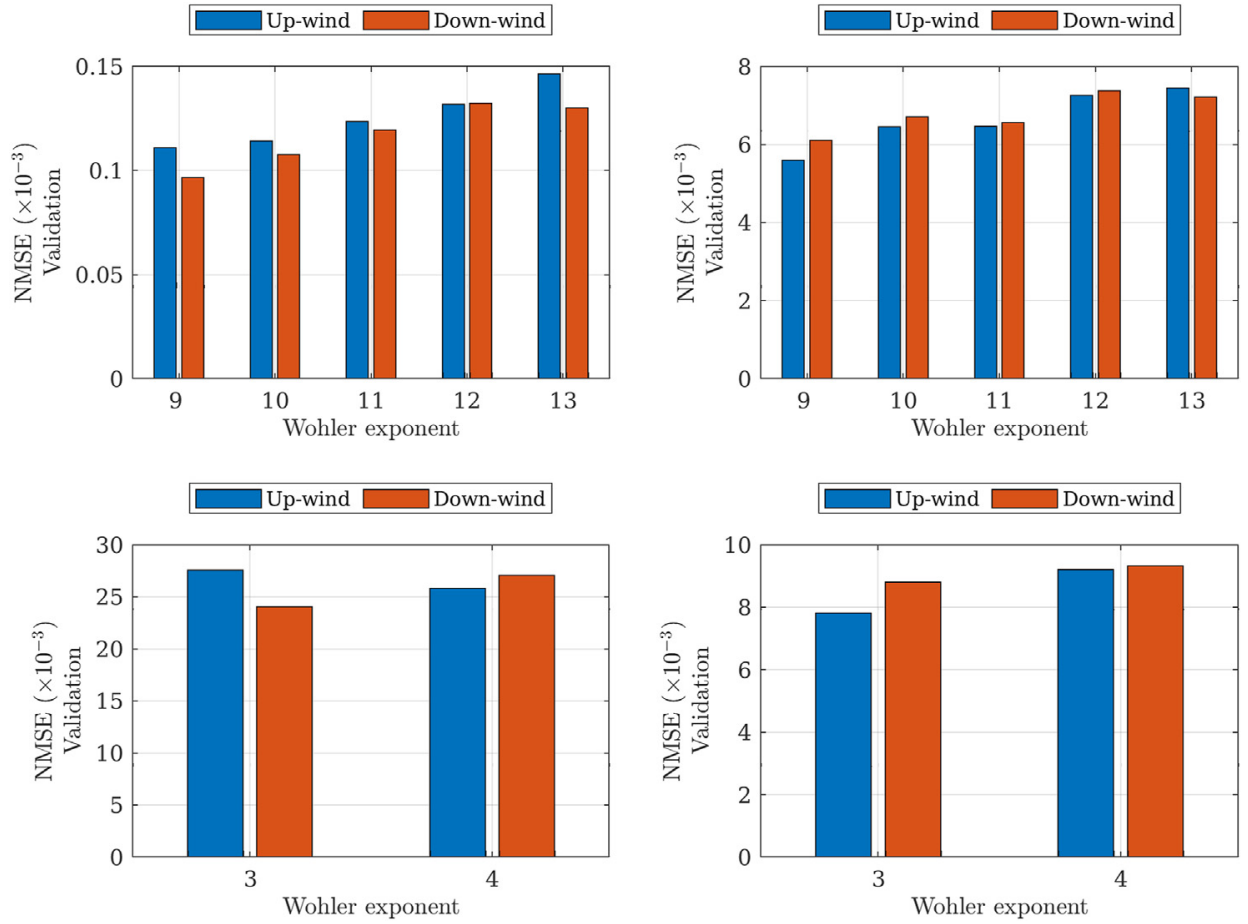

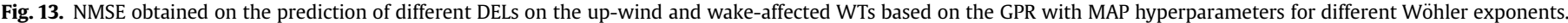
Wake-affected WT located at 11 rotor diameters. Top left: blade edgewise; top right: blade flapwise; bottom left: tower side-to-side; bottom right: tower fore-aft.
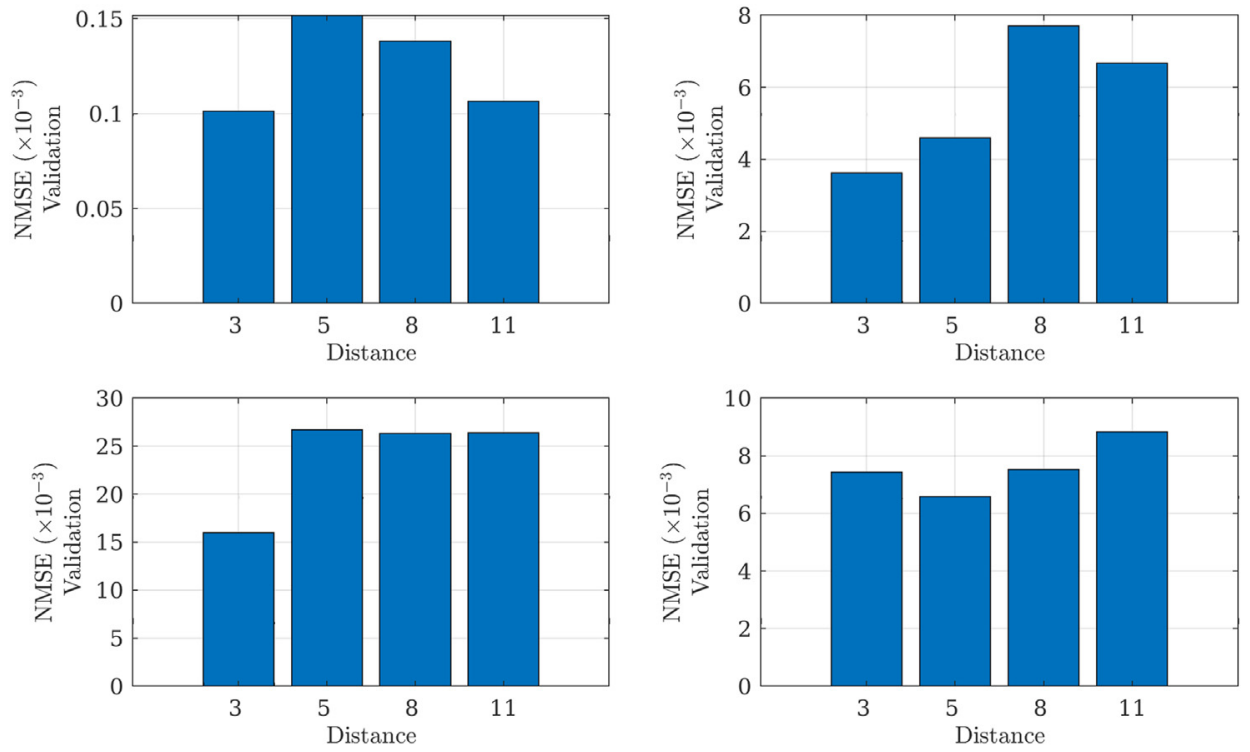

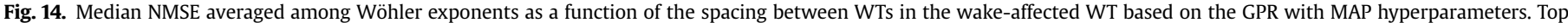
left: blade edgewise; top right: blade flapwise; bottom left: tower side-to-side; bottom right: tower fore-aft.

exponent following in ranking. On the other hand, the horizontal inflow skewness has a much lesser influence on the variation of the DELs. Considering that the range of the variables is normalized to the range $[0,1]$, then the horizontal inflow skewness turns out to be inconspicuous in the DELs. This result can be contrasted with the response slices displayed in Fig. 10 for the wind turbine in the upwind position. In effect, the wind speed seems to have the most complex influence in the DELs, with the turbulence intensity and shear exponent introducing a less complex and almost linear variation, while the horizontal inflow skewness seems to have a very reduced influence in the DELs. On the other hand, contrasting the increments obtained for different Wöhler exponents, it appears that this variable makes no difference on the sensitivity of DELs to input variables.

A similar interpretation can be provided for the loads in the blade flapwise direction, and in the tower side-to-side and fore-aft directions. In the case of the blade flapwise direction and tower fore-aft direction, the DELs are also sensitive to wind speed, turbulence and shear exponent, while insensitive to the horizontal inflow skewness. In contrast, the loads in the tower side-to-side 

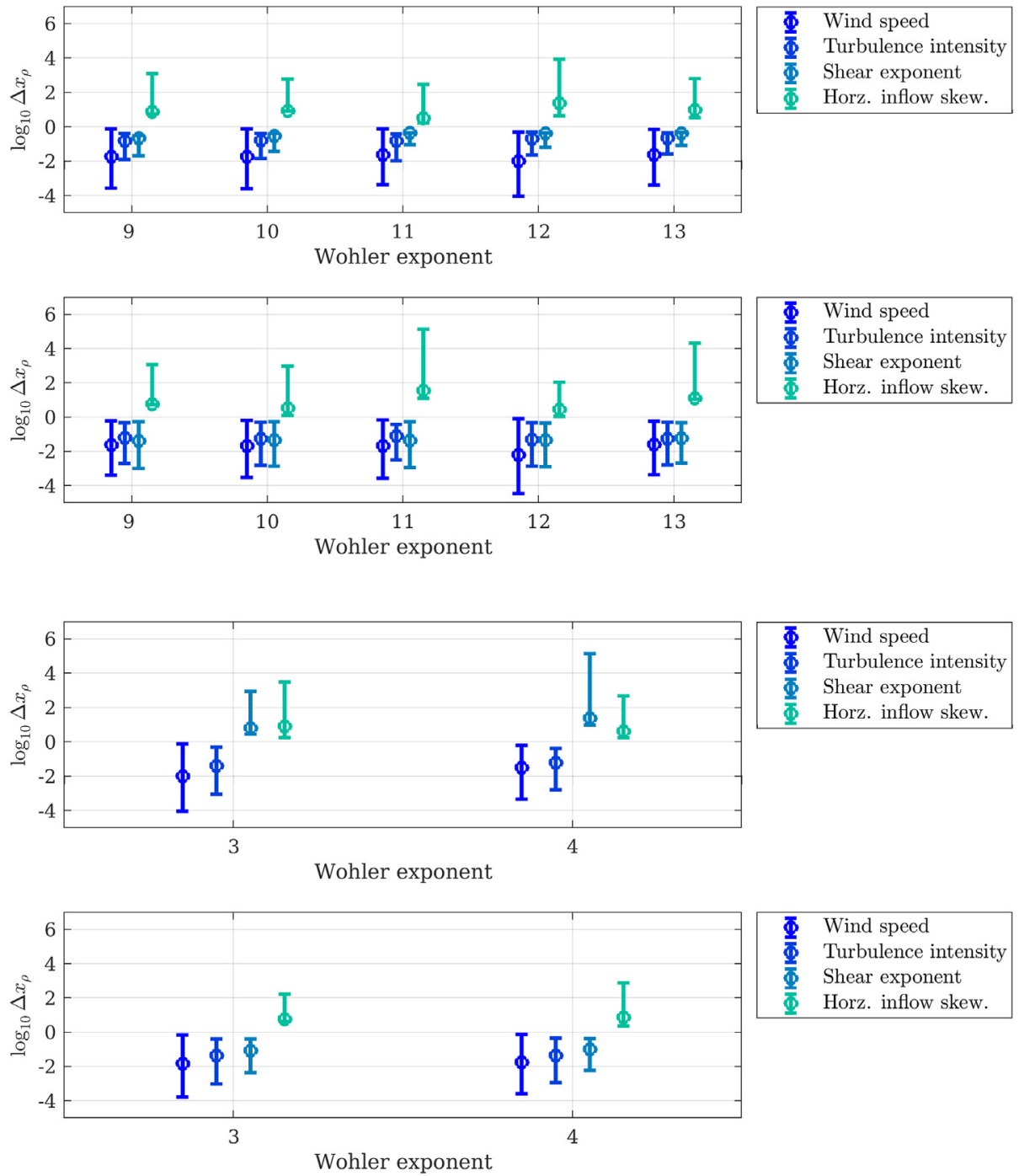

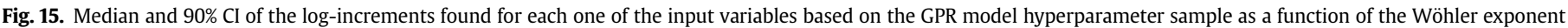
for different components of the up-wind WT. From top to bottom: Blade edgewise loads, blade flapwise loads, tower side-to-side loads, tower fore-aft loads.

direction are insensitive to both the shear exponent and horizontal inflow skewness. In all cases, the Wöhler exponent has no influence on the sensitivity of the DELs to input variables.

With increasing wind speed, the rotor RPM increases linearly below rated wind speed. This invariably results in increased number of fatigue load cycles affecting the blade edgewise loads (most of which are driven by the weight of the blade), which explains the influence of mean wind speed on blade edgewise DEL (more so than turbulence and shear). Similarly, the mean thrust loading increases with increasing mean wind speed, which directly influences the flapwise loads. This is explained by the fact that at higher Wöhler exponents, small variations in the mean loads have a disproportionate influence on the DEL. Turbulence introduces stochasticity to the load ranges, while shear introduces cyclic variations to the load ranges over one blade rotation, which explains their influence on blade and thrust driven tower fatigue loads in the fore-aft direction (e.g. turbulence driving low cycle fatigue which is critical on welded steel components such as the tower). Further conclusion is that the results show marginal sensitivity to errors in the Wöhler exponent.

Fig. 16 provides a similar analysis, this time on the loads of the wake-affected WT as a function of the spacing between WTs, with the Wöhler exponent set to $m=9$ in the case of the blade loads and $m=3$ in the case of the tower loads. For the blade edgewise direction loads, the spacing seems to bear no influence on the sensitivity. However, it now appears that the horizontal inflow skewness has an effect in the DELs of the wake-affected WT measured in the blade edgewise direction. In the case of the blade flapwise loads, the spacing between WTs seems to influence the DELs, but not significantly. For the tower loads, the sensitivity to the shear exponent and horizontal inflow skewness appears to vary with the spacing between WTs. Particularly, for certain spacing the shear exponent has an effect on the loads while for others it does not. Finally, the effect of wind speed and turbulence intensity in the loads appears to be stable in the wake-affected WT regardless of the spacing to the up-wind WT.

\subsubsection{Comparison of up-wind and down-wind DELs}

The obtained GPRs are also used to determine the difference of the loads in the up-wind and wake-affected WTs. Pointwise differences can be evaluated by directly comparing the DEL predictions of the GPRs of the up-wind and down-wind WTs on a test input point $\boldsymbol{x}_{*}$, simply as: 

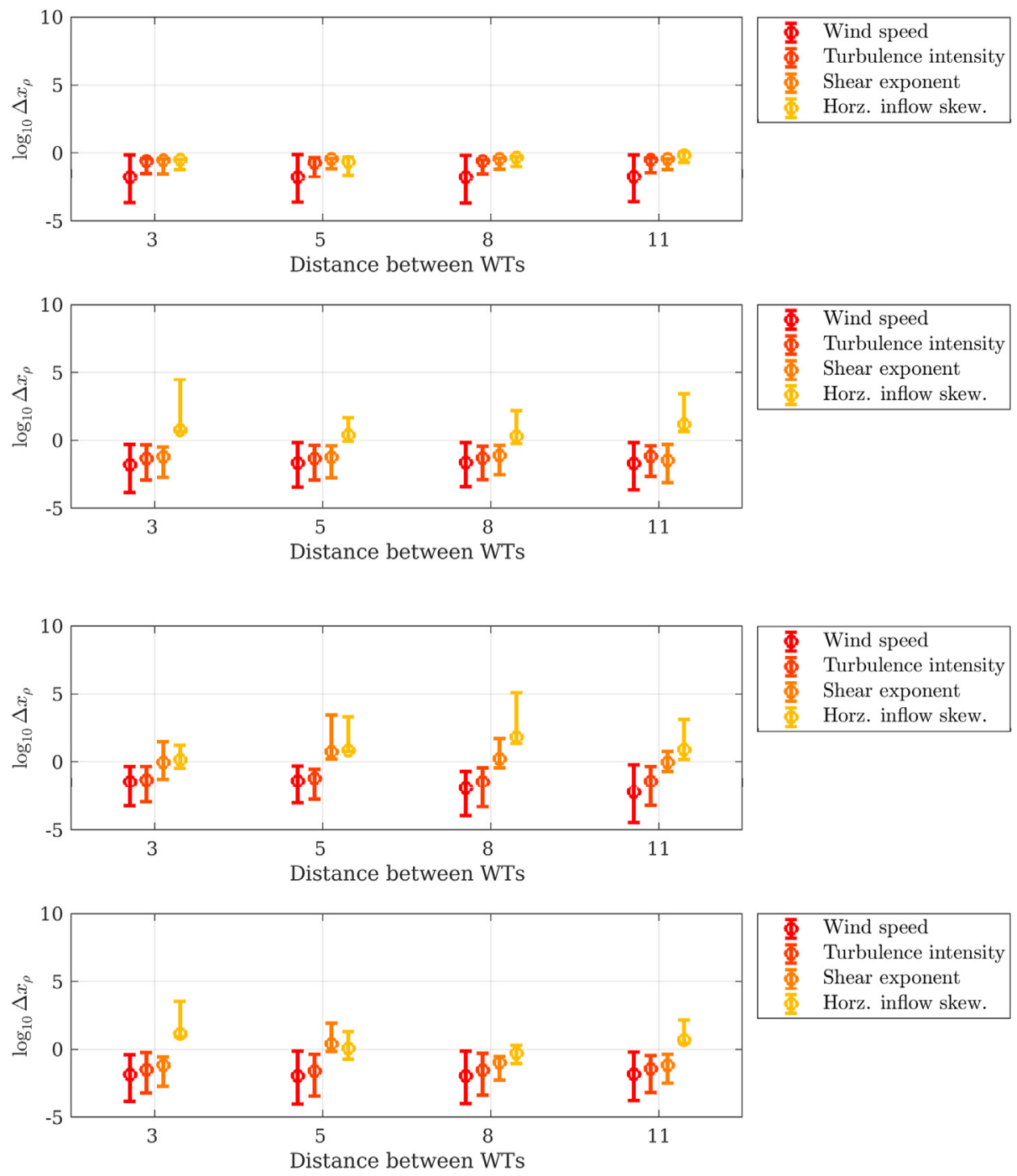

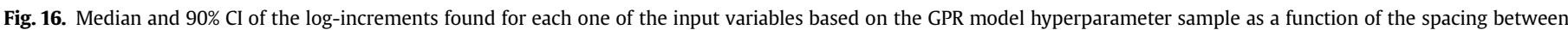
WTs for different components of the wake-affected WT. From top to bottom: Blade edgewise loads, blade flapwise loads, tower side-to-side loads, tower fore-aft loads.

$\Delta f(\boldsymbol{x} *):=\bar{f}_{a}\left(\boldsymbol{x}_{*}\right)-\bar{f}_{b}(\boldsymbol{x} *)$

where $\bar{f}_{a}\left(\boldsymbol{x}_{*}\right)$ and $\bar{f}_{b}\left(\boldsymbol{x}_{*}\right)$ are the GPR predictive mean of the respective sensor in the up-wind and down-wind WTs. In this sense, DEL differences are calculated on a grid of input parameter values to compose hypersurfaces. Likewise, pointwise K-L divergences are calculated as in Eq. (20) on a grid of input parameters values, to construct a surface of pointwise K-L divergences. Fig. 17 shows the surfaces obtained on the case of the DELs in the blade edgewise direction for different values of wind speed and turbulence intensity, with the remaining input variables set to a fixed value (shear exponent: 0.3; horizontal inflow skewness: $2.95^{\circ}$, Wöhler exponent: 9, spacing between WTs: 11 rotor diameters).

The DEL point-wise difference shown in the left frame of Fig. 17 indicate that the predicted DELs tend to be higher in the up-wind wind turbine on low wind speeds, while lower on higher wind speeds. The point-wise K-L divergences shown in the right frame of Fig. 17 demonstrate that higher differences in DEL predictions are found on low turbulence intensity values on almost the complete range of wind speeds. It is noted that although the point-wise difference on low wind speeds appears to be significant, the increased predictive variance on that region due to a lower number of training points makes the difference on the mean be overwhelmed by the respective variance, and thus the corresponding K$\mathrm{L}$ divergences evaluated on the same region are not as significant. On the contrary, larger $\mathrm{K}$-L divergences are found on regions with a larger population of training samples, which makes these results more reliable.

The isolated effect of a single input variable on the K-L divergence can be obtained by marginalizing with respect to the remaining input variables. This is done in Fig. 18, where marginalized K-L divergences, according to Eq. (22), are calculated for the wind speed, turbulence intensity, shear exponent and horizontal inflow skewness for the DELs in the blade edgewise direction for different spacing between WTs. Regarding to the wind speed, the main difference appears for wind speeds between 5 and $15 \mathrm{~m} / \mathrm{s}$, while at high wind speeds the difference appears to be less pronounced. The DELs obtained on turbulence intensities around 15\% also display the largest differences. Similarly, shear exponents close to 0.5 also introduce large difference on the DELs. Moreover, the horizontal inflow skewness induces larger changes in the DELs as its absolute value increases, as could be expected. The difference is more pronounced as the spacing between WTs is reduced, although 

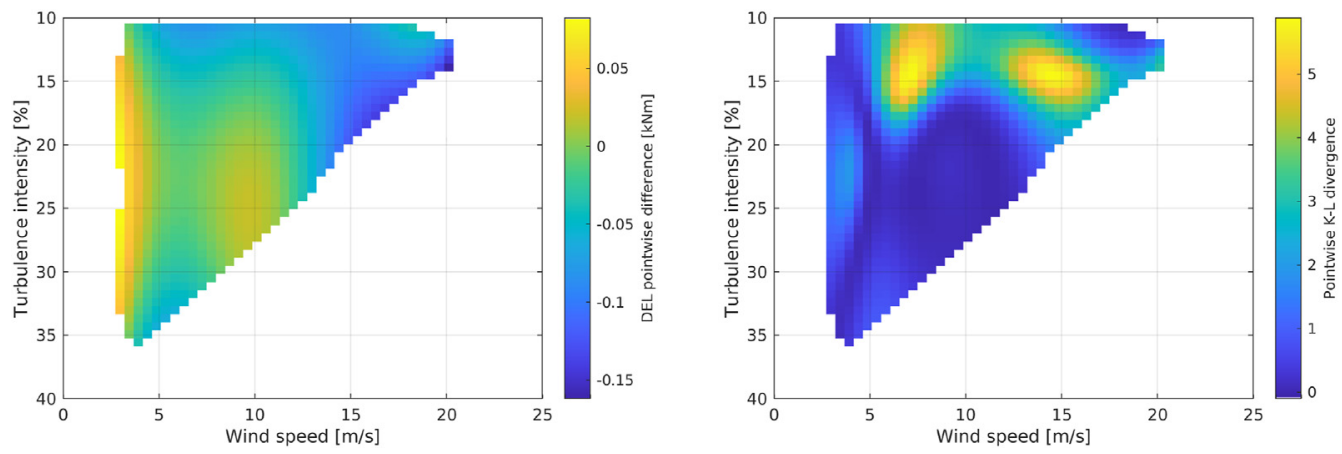

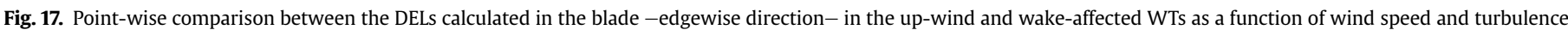

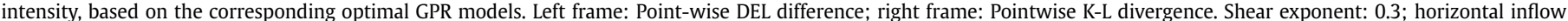
skewness: $2.95^{\circ}$, Wöhler exponent: 9 , spacing between WTs: 11 rotor diameters.
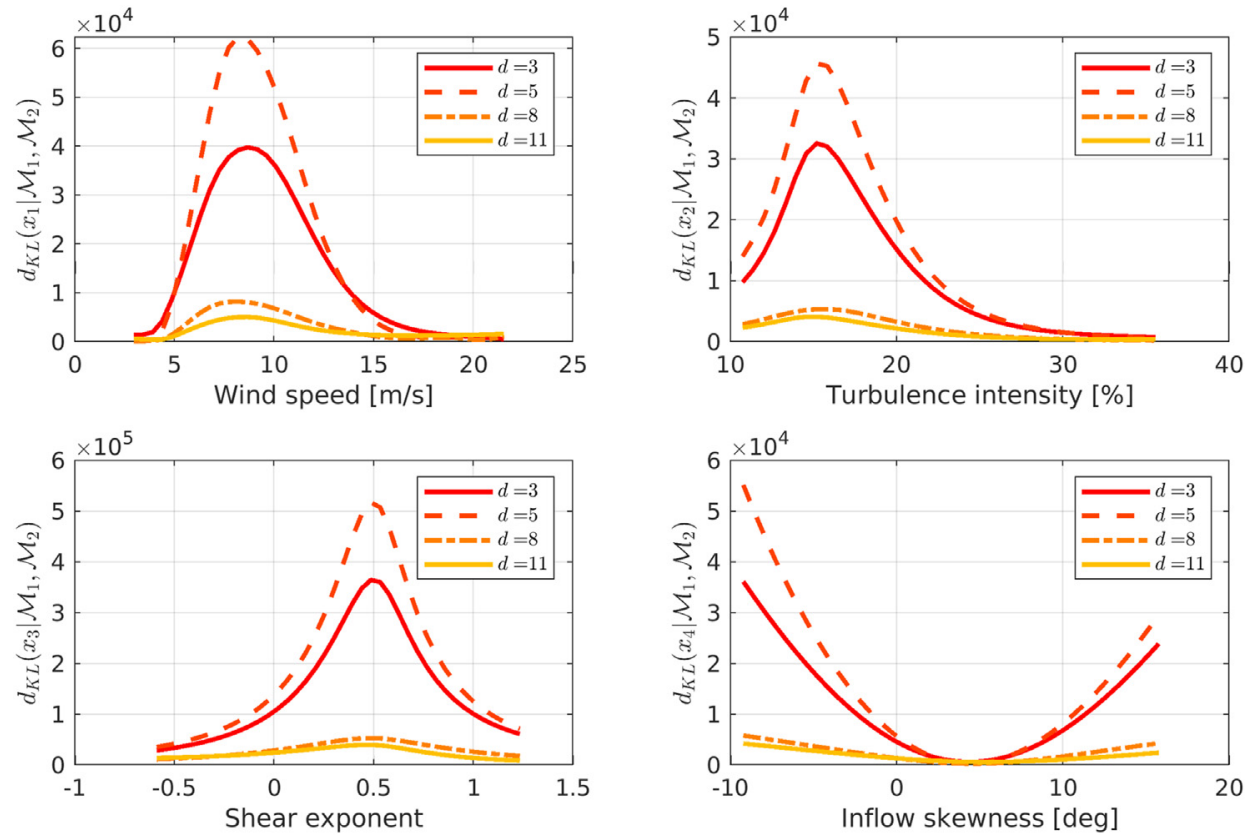

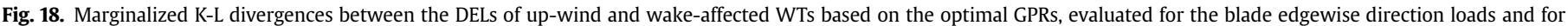
different WT spacings. Wöhler exponent: 9.

the highest difference is found when the spacing is 5 rotor diameters. This tendency is consistent on all the input variables. In section 3.1, we established that the DEL of the blades (composite materials) are more affected by the mean wind speed versus turbulence. When the spacing is 3 or 5 rotor diameters, the wakeaffected wind turbine experiences pronounced increase in inflow turbulence and drop in mean wind speed. Furthermore, when the spacing is 3 or $5 \mathscr{D}$ the large scale effects of wake meandering dominates the small scale mixing in the wake. According to Ref. [31] wake meandering is fully developed (maximal) at around $4 \mathscr{D}$ downstream compared to $3 \mathscr{D}$. A more developed wake meandering induces larger loads variations on the downwindturbine, which possibly explains why we find the highest divergence when the spacing is at $5 \mathscr{D}$ and not at $3 \mathscr{D}$. Furthermore, the point of "DWM model validity" is typically assumed to begin at 3 diameters, where the near-wake pressure field and swirl velocity are close to zero as modelled by the DWM model [16] which might have added some uncertainties to our loads simulations for the $3 \mathscr{D}$ spacing case. Hence, strong wake meandering under convective condition could account for the high KL divergence at $5 \mathscr{D}$ spacing, and the rapid decrease with the growth of spacing is due to the fast recovery of the velocity deficit in the wind turbine wake.

A summary of the global K-L divergence, calculated as in Eq. (21), for the DELs in the blade edgewise and flapwise directions and in the tower base in the fore-aft and side-to-side directions for different WT spacings and Wöhler exponents are provided in Fig. 19. The tendency of the DELs on the blade edgewise direction -top left frame, Fig. 19- follows that described for the marginalized $\mathrm{K}$-L divergences in Fig. 18, namely, higher differences are found for short WT spacings. The tendency follows on the different Wöhler exponents with some variability. On the other hand, in the blade flapwise direction, the DELs seem to have higher differences on shorter spacings but also on higher, while the difference is not as marked on the intermediate spacing values. The difference seems to increase as the Wöhler exponent increases in value. The DELs in the tower base fore-aft direction appear to have higher differences on short WT spacings, but this seems to not be the case for the DELs in the side-to-side direction, which appear to have a less predictable relation to the spacing between WTs. 

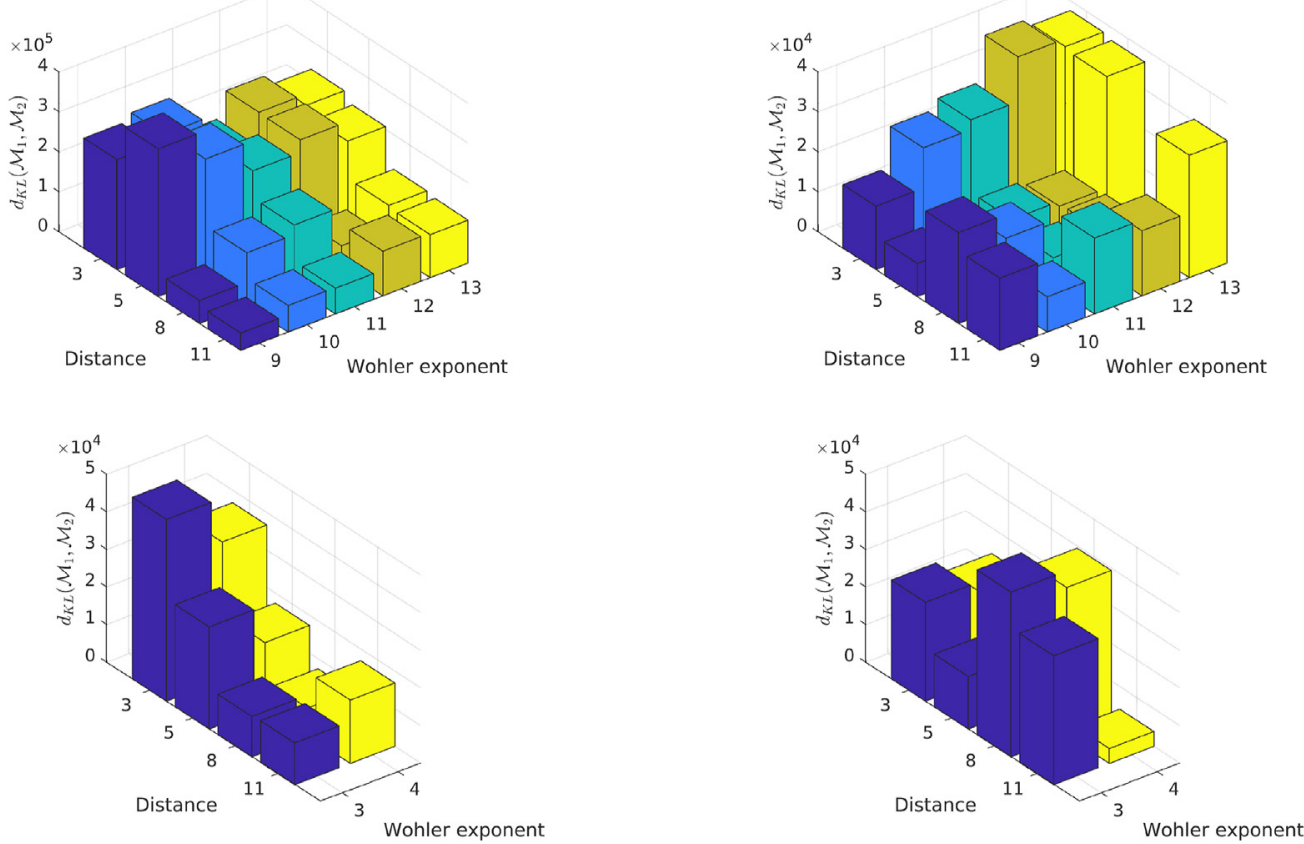

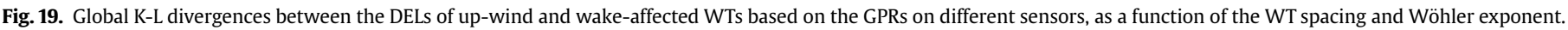
Top-left: blade edgewise direction; top-right: blade flapwise direction; bottom-left: tower fore-aft direction; bottom-right: tower side-to-side direction.

\subsection{Prediction of DELs in the wake-affected WT from remotely measured variables}

In this second analysis, a more realistic scenario, where a measurements from a fully instrumented WT (here the up-wind WT) are used to estimate the DELs in a secondary WT (the wakeaffected WT). The objective of this analysis is to assess the feasibility of load prediction on a secondary WT based on measurements from a fully instrumented one. To this end, different scenarios corresponding to different input variable combinations are considered, as summarized in Table 6 . Scenario 1 corresponds to a still idealized case, where all the wind features of the up-wind WT are available (speed, turbulence intensity, shear exponent and horizontal inflow skewness). Scenarios 2 and 3 reflect more realistic cases, where a few elementary wind field properties of the upwind WT are available (wind speed and turbulence) along with the loads on the blade of the same WT. Finally, scenario 4 corresponds to the case when only loads from the up-wind WT are used to predict loads in the wake-affected WT. The last three scenarios may conform to the conditions of actual wind parks, where one or a few WTs may be fully instrumented, and the objective is then to predict the loads from fully instrumented WTs to adjacent ones.

As in the previous analysis, independent GPRs are built for the DELs measured on the blade root and tower base of the wakeaffected WTs at different spacings and with different Wöhler

\section{Table 6}

List of variables measured in the up-wind WT used for prediction of DELs in the wake-affected WT for each one of the remote prediction scenarios.

\begin{tabular}{ccccccccc}
\hline Scen. & \multicolumn{7}{l}{ Input variables (Measured only in up-wind WT) } \\
\hline & $U_{\text {up }}$ & $\sigma_{\text {up }}$ & $\alpha_{\text {up }}$ & $\psi_{\text {up }}$ & DEL & & \\
1 & $\checkmark$ & $\checkmark$ & $\checkmark$ & $\checkmark$ & & $D E L_{B F W}$ & $D E L_{T F A}$ & $D E L_{T S S}$ \\
2 & $\checkmark$ & $\checkmark$ & & & $\checkmark$ & $\checkmark$ & & \\
3 & $\checkmark$ & & & & $\checkmark$ & $\checkmark$ & & \\
4 & & & & & $\checkmark$ & $\checkmark$ & $\checkmark$ & $\checkmark$ \\
\hline
\end{tabular}

exponents. Moreover, input and output space normalization is performed as in Sec. 5.1, while hyperparameter optimization is carried out with the Bayesian approach based on MH sampling, whose details are provided in Sec. 5.1.1 and in Table 5.

A comparison of the performance, in terms of the NMSE (Eq. (24)), obtained on the prediction of DEL in the wake-affected WT based on local and up-wind EOP measurements is presented in Fig. 20. The performance is presented as a function of the spacing between WTs with the Wöhler exponent set to 9 for the blade loads and 3 for the tower loads. The overall predictive ability of the local and remote prediction scenarios is quite similar in all the cases. In the case of the blade DELs shown in Fig. 20a-(b), the best performance is obtained in scenarios 2 and 3 , which include the wind speed and the blade loads of the up-wind WT, while the remaining scenarios seem to follow closely. Otherwise, in the case of the tower loads, the error performance in all the cases is roughly the same. These results seem to indicate that it is feasible to predict the loads in the wake-affected wind turbine based on measurements obtained from an adjacent WT, although in a practical case it should be necessary to include variables such as the wind direction into the regression model. According to Fig. 20 the lowest load predictions NMSE of the wake-affected wind turbine generally occur when the spacing between the two turbines is $11 \mathscr{D}$. It is simply due to the recovery of the velocity deficit and diffusion of the wake meandering rendering equivalent the aerodynamic loading in the upwind and wake-affected wind turbines. We observe that an effective approach to reduce the load prediction errors on the wake-affected wind turbines when spacing is in the range $3-5 \mathscr{D}$ is to introduce as input to the GPR predictive model some information about the inflow (e.g. mean wind speed) in addition to information about the load condition of the up-wind WT (e.g. blade load). The reason is that those two categories of variables are at the basis of the wake formation and transport that will affect the loads on the down-wind WT.

A global sensitivity analysis of all variables included in the remote prediction scenarios, in terms of the log-increments defined 
(a)

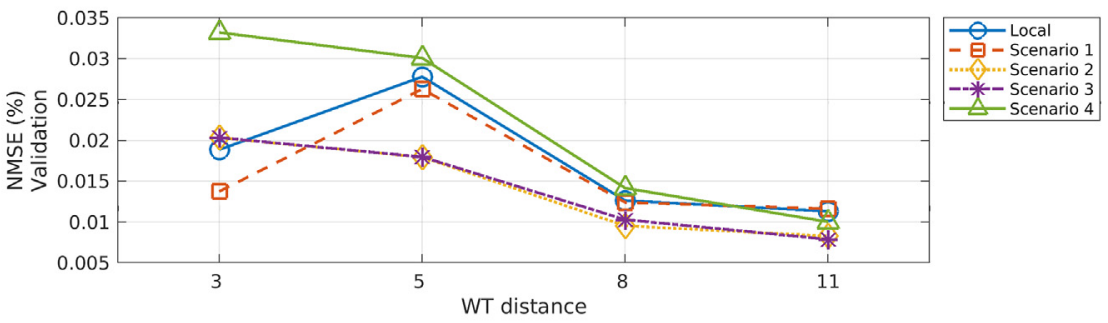

(b)

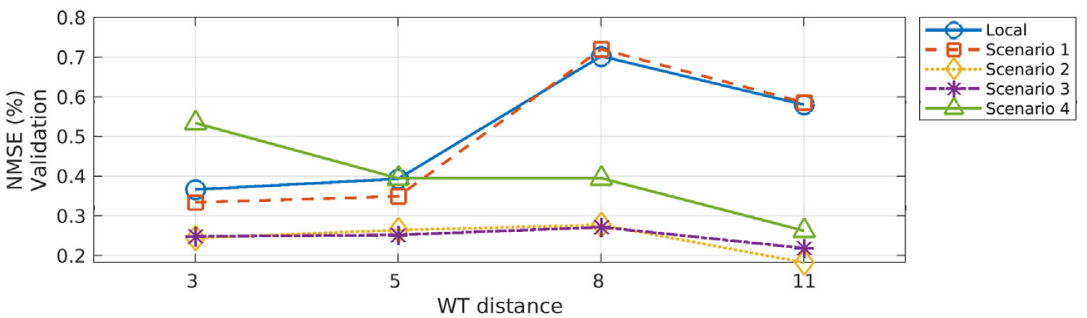

(c)

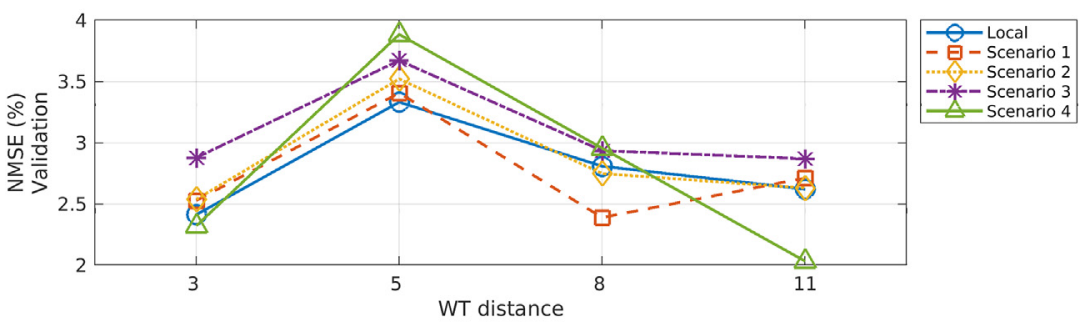

(d)

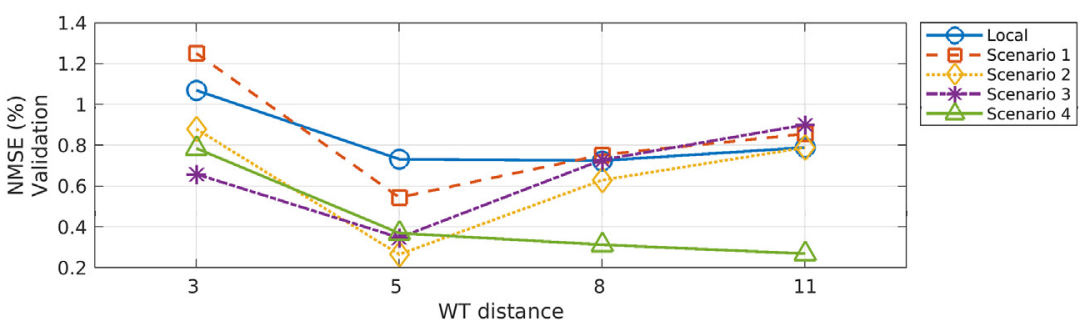

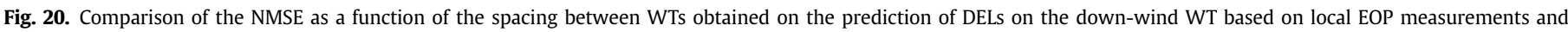

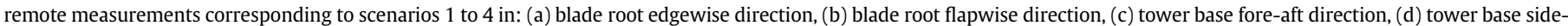
to-side direction.

in Sec. 4.4, is performed with the aid of the MC samples of the hyperparameter posterior obtained at each scenario. The resulting median and 90\% confidence intervals are displayed in Fig. 21 for each one of the sensors in the blade and tower of the wake-affected WT. The cases where error bars are not visible correspond to variables not used in the respective scenario. Most notably, the distribution of the log-increments appears to coincide among the different scenarios. The exception corresponds to the DELs of the blade root flapwise direction, where the sensitivity to wind speed and up-wind DELs changes as other variables are introduced in the analysis. In general, it can be observed that the wind speed, turbulence intensity and the DELs in the blade of the up-wind turbine seem to be better predictors of the loads in the wake-affected WT.

\section{Concluding discussions and outlook}

We demonstrated how a GPR-based model can be built and calibrated to effectively predict the loads on a wake affected wind turbine either from local or remote wind field sensors and/or loads sensors in adjacent WTs in a wind farm. The maximum prediction error, obtained in the tower base DELs in the fore-aft direction, results in less than $4 \%$ for the simulations investigated herein. In the case of the blade DELs, the overall prediction error is less than $1 \%$.
Although simple, the test configuration allows us to explore sensor selection (scenario) with the aim to minimize the prediction NMSEs according to the spacing between turbines. According to Table 6 scenario 4 tends to yield the lowest NMSE of the predictions for simultaneously the blade-root and tower base loads on the wakeaffected WT when the spacing is $11 D$, but returns the highest prediction error for tower fore-aft when WT spacing is $5 D$. It turns out that introducing wind inflow RVs as further input to the GPR model (as in scenarios 2 and 3 ) tends to reduce the predictions NMSE on the wake-affected WT when the spacing is $5 D$ or less.

The sensitivity analysis carried out on the GPR hyperparameters and the K-L divergence reveals that the wind speed and turbulence intensity are the main drivers of the blade root and tower base loads in a WT receiving the freestream inflow as well as in a wake affected wind turbine (see Fig. 15). The wind shear affects the blade loads to a lesser extent on the upwind wind turbine, while the horizontal inflow skewness has no noticeable effects on the upwind WT. The wind shear has an increased significance on the wakeaffected WT (see Fig. 16), while the horizontal inflow skewness marginally affects the blade DELs in the edgewise direction and tower side-side direction.

We stress that the estimations of the GPR model are trustworthy only within the bounds of the variable space used for model 
(a)

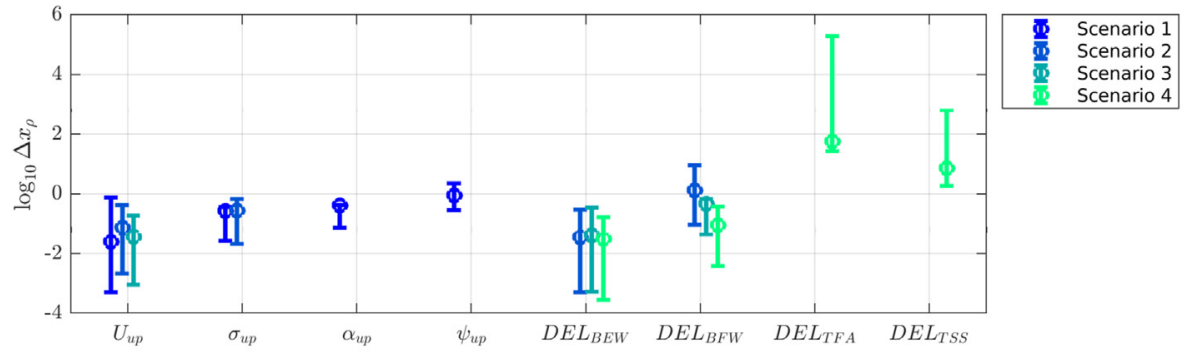

(b)

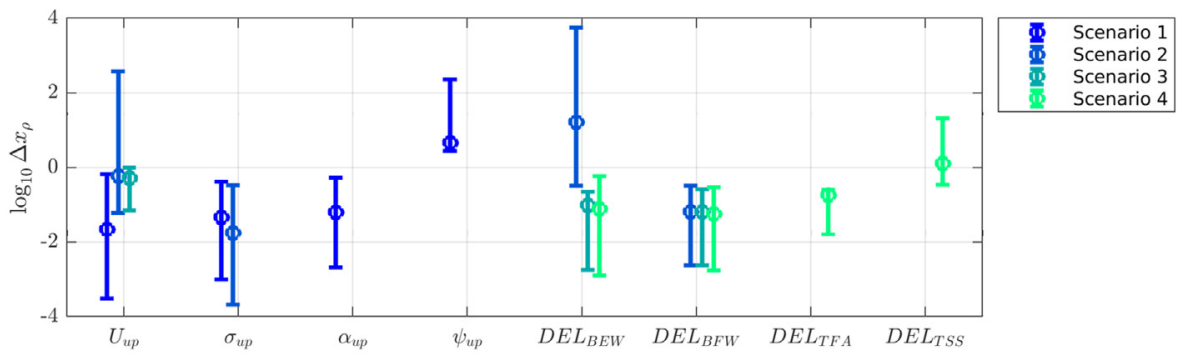

(c)

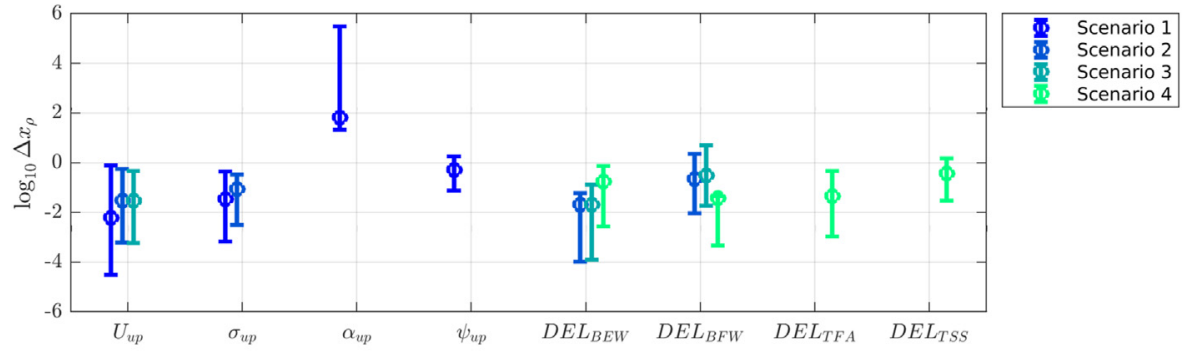

(d)

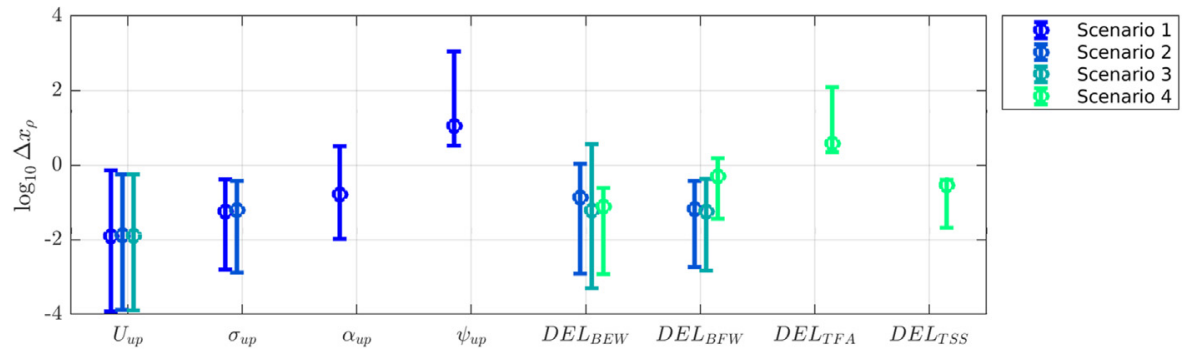

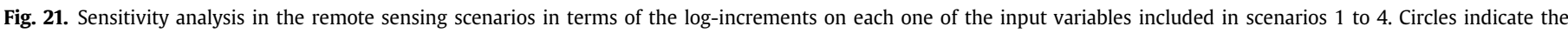

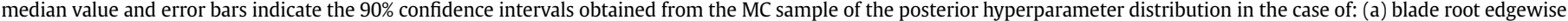
direction, (b) blade root flapwise direction, (c) tower base fore-aft direction, (d) tower base side-to-side direction.

calibration; any extrapolation is either not possible or may lead to unpredictable/wrong results. It is therefore important to ensure that the distributions used for the fatigue load diagnostics are not outside the bounds of validity of the trained GPR model.

We note that we chose the average wind speed, turbulence, wind shear and wind inflow skewness as the RVs that describe the ambient freestream wind inflow conditions. There may be additional random variables that are equally suitable for the purpose, such as the atmospheric stability that has a complex dependence on the wind speed, turbulence, and wind shear. While combinations of the aforementioned variables usually correspond to certain stability conditions and can be used as a proxy, it may be more convenient to directly include stability as a variable as demonstrated in Refs. [25,29].

Outlook: We purposely initiate from a simulated experiment adopting a simplified, yet educative, configuration of two wind turbines. In next steps, we intend to look into scalability and generalization of GPR with Bayesian learning for larger predictive tasks in arbitrary wind farm layout, possibly harnessing recent progress in scalable GPs, e.g. scalable GPR algorithm, namely, kernel interpolation for scalable structured Gaussian processes (KISS-GP) along with massively scalable GP (MSGP) [32].

In the past the DWM model performance has been validated against numerous measured load data sets, while in the present study, a surrogate model-based approach is validated only against the DWM-FAST model predictions. However, direct validation of the performance of the proposed GPR approach against measured loads data was not currently possible. This is an obvious potential topic for future research, which we are actively working towards.

We elucidated how using the DELs one can quantify significant differences in the dynamic response between WTs receiving freestream wind and those wake-affected WTs. These differences are more apparent in the mid-range of wind speeds and turbulence intensity (wind speed from 5 to $15 \mathrm{~m} / \mathrm{s}$ and turbulence intensity from 10 to 25\%). This result suggests that the fatigue life on a WT subject to freestream inflow can be quite distinct from that of a WT operating in waked condition. Nonetheless, as wind field parameters are the main drivers of short-term DELs, it is difficult to predict 
which of the cases will lead to a long-term reduced fatigue life on a given structural component. Nonetheless, regression models such as the GPR presented here can be used as surrogates of a WT in a wind park and perform MC simulations to estimate fatigue life. This would be a subject of study in a future work. Furthermore, in practice, the long term material degradation of various structural components of the up-wind WT differs from that of the wakeaffected WT. So as time passes, the GPR model predictions will gradually diverge from the real short-term DEL experienced by the wake-affected wind turbine. Our GPR approach will have to be modified in order to capture such multi-valued and timedependent relationships using some form of overlapping mixture of probabilistic GPR models with recurrent model parameters updates.

Although the WT set-up presented in this work is a rather simplified approximation of actual layouts in a wind park, it has provided many insights on the random variables influencing the DELs on a wake-affected WT. This set-up also allowed for testing of various combinations of wind field and loads sensors in the upwind WT as input for predicting the loads on a wake-affected wind turbine. In an actual wind park, wind direction may also greatly determine the DELs in a wake-affected WT. Namely, a wakeaffected WT can change from a fully-waked condition to a freestream condition according to the wind direction and the position of adjacent WT. Also, the severity and aggregation of the wake(s) can change according to the number of adjacent WTs from a specific wind direction. This case will also be considered in a future work.

Finally, in section 3 we attempted to provide a qualitative interpretation of the simulations results. Deploying probabilistic deep machine learning algorithms could be one way to automatically and more systematically learn such complex relationships and dependence structures in high dimensional spaces. These algorithms however will potentially lack the interpretability of the underlying physical process but would make up for it in inferring functional relationships which are crucial in virtual diagnostics of wake affected WT from adjacent WT. The consequence is the realization that a physics-informed deep learning approaches might be the way forward. This case is in fact an active area of research of ours.

\section{CRediT authorship contribution statement}

Luis David Avendaño-Valencia: Data curation, Writing - original draft, Conceptualization, Methodology, Investigation, Visualization, Software, Writing - review \& editing. Imad Abdallah: Data curation, Writing - original draft, Conceptualization, Methodology, Investigation, Visualization, Software, Writing - review \& editing. Eleni Chatzi: Funding acquisition, Supervision, Writing - review \& editing.

\section{Declaration of competing interest}

The authors declare that they have no known competing financial interests or personal relationships that could have appeared to influence the work reported in this paper.

\section{Acknowledgments}

The authors acknowledge the support of the European Research Council via the ERC Starting Grant WINDMIL (ERC-2015-StG \#679843) on the topic of Smart Monitoring, Inspection and LifeCycle Assessment of Wind Turbines. The authors would further like to acknowledge the support the ERC Proof-of-Concept Grant ERC-2018-PoC WINDMIL RT-DT (funded under H2020-EU.1.1., grant agreement ID 825833). The authors would like to thank OSMOS Hellas SA for the granting of graphic material appearing in Fig. 1.

\section{References}

[1] 61400-1. Wind Turbines, Part 1 Design Requirements, International Electrotechnical Commission, 2005. Technical Report IEC 61400-1:2005(E).

[2] I. Abdallah, V. Dertimanis, H. Mylonas, K. Tatsis, E. Chatzi, N. Dervili, K. Worden, Eoghan Maguire, Fault Diagnosis of Wind Turbine Structures Using Decision Tree Learning Algorithms with Big Data, 2018.

[3] I. Abdallah, C. Lataniotis, B. Sudret, Parametric Hierarchical Kriging for MultiFidelity Aero-Servo-Elastic Simulators - Application to Extreme Loads on Wind Turbines, Probabilistic Engineering Mechanics, 2018.

[4] I. Abdallah, A. Natarajan, J.D. Sörensen, Impact of uncertainty in airfoil characteristics on wind turbine extreme loads, Renew. Energy 75 (2015) 283-300.

[5] Anis Ben Abdessalem, Nikolaos Dervilis, David J. Wagg, Worden Keith, Automatic kernel selection for Gaussian processes regression with approximate bayesian computation and sequential Monte Carlo, Frontiers in Built Environment 3 (2017) 52.

[6] Luis David Avendaño-Valencia, N. Eleni, Chatzi. Multivariate gp-var models for robust structural identification under operational variability, Probabilist. Eng. Mech. 60 (2020) 103035.

[7] Luis David Avendaño-Valencia, Eleni N. Chatzi, Ki Young Koo, M. James, W. Brownjohn, Gaussian process time-series models for structures under operational variability, Frontiers in Built Environment 3 (2017) 69.

[8] Luis David Avendaño-Valencia, Eleni N. Chatzi, Dmitri Tcherniak, Gaussian process models for mitigation of operational variability in the structural health monitoring of wind turbines, Mech. Syst. Signal Process. 142 (2020) 106686.

[9] Christopher M. Bishop, Pattern Recognition and Machine Learning (Information Science and Statistics), 1 edition, Springer, 2007.

[10] Nicolai Cosack, Fatigue Load Monitoring with Standard Wind Turbine Signals, PhD thesis, Universität Stuttgart, Germany, 2010.

[11] Rick Damiani, Greg Hayman, The Unsteady Aerodynamics Module for FAST 8, National Renewable Energy Laboratory, NREL/TP-5000-66347, 2019. Technical report.

[12] N. Dimitrov, M.C. Kelly, A. Vignaroli, J. Berg, From wind to loads: wind turbine site-specific load estimation with surrogate models trained on high-fidelity load databases, Wind Energy Science 3 (2) (2018) 767-790.

[13] Nikolay Dimitrov, Surrogate models for parameterized representation of wake-induced loads in wind farms, Wind Energy 22 (10) (2019) 1371-1389.

[14] Nikolay Dimitrov, Anand Natarajan, From SCADA to lifetime assessment and performance optimization: how to use models and machine learning to extract useful insights from limited data, J. Phys. Conf. 1222 (2019), 012032.

[15] N.K. Dimitrov, A. Natarajan, M. Kelly, Model of wind shear conditional on turbulence and its impact on wind turbine loads, Wind Energy 18 (2014) 1917-1931.

[16] Brandon Lee Ennis, Christopher Lee Kelley, and David Charles Maniaci. Dynamic Wake Meandering Model Comparison with Varying Fidelity Models for Wind Turbine Wake Prediction.

[17] Christos Galinos, Nikolay Dimitrov, Torben J. Larsen, Anand Natarajan, Kurt S. Hansen, Mapping wind farm loads and power production - a case study on Horns Rev 1, in: In Journal of physics Conference series, vol 753 of Journal of Physics Conference Series, 2016.

[18] Fabian Grasse, Volker Trappe, Sebastian Thöns, Samir Said, G. De Roeck, G. Degrande, G. Lombaert, G. Müller, Structural health monitoring of wind turbine blades by strain measurement and vibration analysis. Number MS25 Tue, pages 3490-3497, Bundesanstalt fuer Materialforschung und -pruefung (BAM), Berlin, Germany, 2011.

[19] Fabian Grasse, Volker Trappe, Sebastian Thöns, Samir Said, Structural health monitoring of wind turbine blades by strain measurement and vibration analysis, in: G. De Roeck, G. Degrande, G. Lombaert, G. Müller (Eds.), EURODYN 2011 - 8th International Conference on Structural Dynamics (Proceedings), Number MS25 Tue, 2011, pp. 3490-3497.

[20] Hao Yujia, Matthew A. Lackner, Rolf-Erik Keck, Sang Lee, Matthew J. Churchfield, Patrick J. Moriarty, Implementing the dynamic wake meandering model in the nwtc design codes, in: 32nd ASME Wind Energy Symposium, National Harbor, Maryland, Wahington, DC, AIAA, 2014.

[21] A. Iliopoulos, Christof Devriendt, P. Guillaume, D.V. Hemelrijck, C Ontinuous Fatigue Assessment of an Offshore Wind Turbine Using a Limited Number of Vibration Sensors, 2015.

[22] J. Jonkman, M. Buhl, FAST User's Guide, National Renewable Energy Laboratory, NREL/EL-500-38320, 2005. Technical report.

[23] J. Jonkman, S. Butterfield, W. Musial, G. Scott, Definition of a 5-MW reference wind turbine for offshore system development, National Renewable Energy Laboratory, NREL/EL-500-38060, 2009. Technical report.

[24] J.C. Kaimal, J.C. Wyngaard, Y. Izumi, O.R. CotĀ@), Spectral characteristics of surface-layer turbulence, Q. J. R. Meteorol. Soc. 98 (417) (1972) 563-589.

[25] Rolf-Erik Keck, Martin de MarĀ@, Matthew J. Churchfield, Sang Lee, Gunner Larsen, Helge Aagaard Madsen, On atmospheric stability in the dynamic wake meandering model, Wind Energy 17 (11) (2014) 1689-1710.

[26] Rolf-Erik Keck, Dick Veldkamp, Jens Jakob Wedel-Heinen, Forsberg Jan, A Consistent Turbulence Formulation for the Dynamic Wake Meandering 
Model in the Atmospheric Boundary Layer, PhD thesis, Denmark, 2013.

[27] Soo-Hyun Kim, Hyung-Ki Shin, Young-Chul Joo, Keon-Hoon Kim, A study of the wake effects on the wind characteristics and fatigue loads for the turbines in a wind farm, Renew. Energy 74 (2015) 536-543.

[28] Andrew Kusiak, Zijun Zhang, Anoop Verma, Prediction, operations, and condition monitoring in wind energy, Energy 60 (2013) 1-12.

[29] G.C. Larsen, D.R. Verelst, F. Bertagnolio, S. Ott, A. Chougule, In search for a canonical design ABL stability class for wind farm turbines, J. Phys. Conf. 753 (2016).

[30] Gunner Chr Larsen, Jakob Mann, Søren Ott, J.N. Sørensen, V. Okulov, N. Troldborg, Kenneth Thomsen, Torben J. Larsen, R. Mikkelsen, in: Dynamic wake meandering modeling. Number 1607(EN) in Denmark. Forskningscenter Risoe. Risoe-R, Risø National Laboratory, 2007.

[31] J. Liew, A.M. Urbán, S.J. Andersen, Analytical model for the power-yaw sensitivity of wind turbines operating in full wake, Wind Energy Science 5 (1) (2020) 427-437.

[32] Haitao Liu, Y. Ong, Xiaobo Shen, J. Cai, When Gaussian process meets big data: a review of scalable gps, IEEE transactions on neural networks and learning systems (2019). 1807.01065.

[33] H.A. Madsen, K. Thomsen, G.C. Larsen, A new method for prediction of detailed wake loads, in: Thor Sven-Erik (Ed.), Proceedings of IEA Joint Action of Wind Turbines 16th Symposium, NREL, Boulder, Co., USA, 2003, pp. $171-188$.

[34] Milad Memarzadeh, Matteo Pozzi, J. Zico Kolter, Optimal planning and learning in uncertain environments for the management of wind farms, J. Comput. Civ. Eng. 29 (5) (2015), 04014076.

[35] C. Mylonas, I. Abdallah, E.N. Chatzi, Deep unsupervised learning for condition monitoring and prediction of high dimensional data with application on windfarm scada data, in: Robert Barthorpe (Ed.), Model Validation and Uncertainty Quantification, 3, Springer International Publishing, Cham, 2020, pp. 189-196.

[36] Nymfa Noppe, Konstantinos Tatsis, Eleni Chatzi, Christof Devriendt, Wout Weijtjens, Fatigue stress estimation of offshore wind turbine using a kalman filter in combination with accelerometers, in: D. Moens, W. Desmet, B. Pluymers, W. Rottiers (Eds.), Proceedings of ISMA 2018 - International Conference on Noise and Vibration Engineering and USD 2018 - International Conference on Uncertainty in Structural Dynamics, Proceedings of ISMA 2018 International Conference on Noise and Vibration Engineering and USD 2018 International Conference on Uncertainty in Structural Dynamics, 2018, pp. $4847-4855$.

[37] E. Papatheou, N. Dervilis, A.E. Maguire, I. Antoniadou, K. Worden, A performance monitoring approach for the novel lillgrund offshore wind farm, IEEE Trans. Ind. Electron. 62 (10) (2015) 6636-6644.

[38] Junyoung Park, Jinkyoo Park, Physics-induced graph neural network: an application to wind-farm power estimation, Energy 187 (2019) 115883.

[39] Carl Edward Rasmussen, Christopher K.I. Williams, Gaussian processes for Machine Learning. Adaptive Computation and Machine Learning, MIT Press 2006.

[40] Riccardo Riva, Jaime Yikon Liew, Mikkel Friis-Møller, Nikolay Dimitrov, Emre Barlas, Pierre-Elouan Réthoré, Arvydas Beržonskis, Wind farm layout optimization with load constraints using surrogate modelling, J. Phys. Conf. (4) (2020)

[41] Christian P. Robert, George Casella, Monte Carlo Statistical Methods, second ed., Springer, 2004.

[42] Andrea Saltelli, Paola Annoni, Ivano Azzini, Francesca Campolongo, Marco Ratto, Stefano Tarantola, Variance based sensitivity analysis of model output. design and estimator for the total sensitivity index, Comput. Phys. Commun. 181 (2) (2010) 259-270.

[43] L. Schröder, N.K. Dimitrov, D.R. Verelst, A surrogate model approach for associating wind farm load variations with turbine failures, Wind Energy Science 5 (3) (2020) 1007-1022.

[44] Laura Schröder, Nikolay Krasimirov Dimitrov, David Robert Verelst, John Aasted Sørensen, Wind turbine site-specific load estimation using artificial neural networks calibrated by means of high-fidelity load simulations, J. Phys. Conf. 1037 (2018).

[45] Jens N. Sørensen, Robert Mikkelsen, Sasan Sarmast, Stefan Ivanell, Henningson Dan, Determination of wind turbine near-wake length based on stability analysis, J. Phys. Conf. 524 (jun 2014), 012155.

[46] Federico Tagliatti, Investigation of Wind Turbine Fatigue Loads under Wind Farm Control: Analysis of Field Measurements, Master's Thesis, Delft University of Technology, the Netherlands, 2019.

[47] Konstantinos Tatsis, Eleni Chatzi, Eliz-Mari Lourens, Reliability prediction of fatigue damage accumulation on wind turbines support structures, in: Proceedings of the 2nd International Conference on Uncertainty Quantification in Computational Sciences and Engineering, 2017, pp. 76-89.

[48] Herman Frederik Veldkamp, Chances in Wind Energy, A Probabilistic Approach to Wind Turbine Fatigue Design, PhD Thesis, DUWIND Delft University Wind Energy Research Institute, The Netherlands, 2006.

[49] S. Woo, J. Park, J. Park, Predicting wind turbine power and load outputs by multi-task convolutional lstm model, in: 2018 IEEE Power Energy Society General Meeting (PESGM), 2018, pp. 1-5.

[50] A. Zaher, S.D.J. McArthur, D.G. Infield, Y. Patel, Online wind turbine fault detection through automated scada data analysis, Wind Energy 12 (6) (2009) 574-593. 